\title{
Decay of the 3D Quasilinear Hyperbolic Equations with Nonlinear Damping
}

\author{
Hongjun Qiu ${ }^{1}$ and Yinghui Zhang ${ }^{2}$ \\ ${ }^{1}$ College of Mathematics and Computer Science, Hunan Normal University, Changsha 410081, China \\ ${ }^{2}$ Department of Mathematics, Hunan Institute of Science and Technology, Yueyang 414006, China
}

Correspondence should be addressed to Yinghui Zhang; zhangyinghui0910@126.com

Received 13 February 2017; Accepted 19 April 2017; Published 23 May 2017

Academic Editor: Ming Mei

Copyright (c) 2017 Hongjun Qiu and Yinghui Zhang. This is an open access article distributed under the Creative Commons Attribution License, which permits unrestricted use, distribution, and reproduction in any medium, provided the original work is properly cited.

\begin{abstract}
We investigate the 3D quasilinear hyperbolic equations with nonlinear damping which describes the propagation of heat wave for rigid solids at very low temperature, below about $20 \mathrm{~K}$. The global existence and uniqueness of strong solutions are obtained when the initial data is near its equilibrium in the sense of $H^{3}$-norm. Furthermore, if, additionally, $L^{p}$-norm $(1 \leq p<6 / 5)$ of the initial perturbation is finite, we also prove the optimal $L^{p}-L^{2}$ decay rates for such a solution without the additional technical assumptions for the nonlinear damping $f(v)$ given by Li and Saxton.
\end{abstract}

\section{Introduction}

In this paper, we consider the following 3D quasilinear hyperbolic system with nonlinear damping:

$$
\begin{aligned}
v_{t}-\operatorname{div}(h(v) \mathbf{P}) & =0, \quad x \in \mathbb{R}^{3}, t>0, \\
\mathbf{P}_{t}+\nabla \sigma(v) & =f(v) \mathbf{P}, \quad x \in \mathbb{R}^{3}, t>0,
\end{aligned}
$$

with initial data

$$
\begin{aligned}
(v, \mathbf{P})(x, 0)=\left(v_{0}, \mathbf{P}_{0}\right)(x) \longrightarrow & (\bar{v}, 0), \\
& \text { as }|x| \longrightarrow \infty, \bar{v}>0,
\end{aligned}
$$

where $\sigma^{\prime}(v)<0, h(v)>0, f(v)<0$, and $v>0$. The above system is derived in $[1,2]$ and describes the propagation of heat wave for rigid solids at very low temperatures, below about $20 \mathrm{~K}$. The first equation in (1) comes from the balance of energy, which takes the form

$$
\varepsilon(9)_{t}+\operatorname{div} \mathbf{q}=0,
$$

where $\vartheta>0$ is the absolute temperature, $\varepsilon$ is the internal energy, and $\mathbf{q}$ is the heat flux. The second equation in (1) is the evolution equation for an internal parameter $\mathbf{P}$, which is introduced to account for memory effects of the heat flux. The effect of memory may be considered, for example, as a functional of a history of temperature gradient,

$$
\mathbf{q}=-\alpha(\vartheta) \int_{-\infty}^{t} e^{-b(t-s)} \nabla \vartheta(\mathbf{x}, s) d s, \quad \alpha(\vartheta)>0, b>0 .
$$

By defining

$$
\mathbf{P}=\int_{-\infty}^{t} e^{-b(t-s)} \nabla \mathcal{\vartheta}(\mathbf{x}, s) d s,
$$

then (4) can be equivalently replaced with

$$
\begin{gathered}
\mathbf{q}=-\alpha(\vartheta) \mathbf{P}, \\
\mathbf{P}_{t}=-b \mathbf{P}+\nabla \vartheta .
\end{gathered}
$$

Equation (7), related to (4) via (5), is however linear and does not fully describe the properties of heat propagation in solids (cf. [1-4] and references therein). To improve the model, one may generalize the history dependence of $\mathbf{q}$ by modifying (4) or, as was done in [2], by introducing a suitable nonlinear dependence in (7),

$$
\mathbf{P}_{t}=g_{1}(\vartheta) \nabla \vartheta+g_{2}(\vartheta) \mathbf{P} .
$$


The functions $\alpha, g_{1}$, and $g_{2}$ present in (6) and (8) are material functions. The second law of thermodynamics imposes the restrictions that $\alpha(\vartheta)=\psi_{20} \vartheta^{2} g_{1}(\vartheta)$ and $g_{2}(\vartheta)<0$, where the constant $\psi_{20}$ comes from the Helmholtz free energy $\psi$ which has the form $\psi=\psi_{1}(\vartheta)+(1 / 2) \psi_{20} \vartheta \mathbf{P}^{2}$. We additionally make an assumption that $g_{1}(\vartheta)>0$ (cf. [2]). Combining (3) with (8) gives the following system:

$$
\begin{aligned}
\varepsilon(\vartheta)_{t}-\operatorname{div}(\alpha(\vartheta) \mathbf{P}) & =0, \\
\mathbf{P}_{t}+\nabla \mathscr{G}_{1}(\vartheta) & =g_{2}(\vartheta) \mathbf{P}, \quad \mathscr{G}_{1}^{\prime}(\vartheta)=-g_{1}(\vartheta) .
\end{aligned}
$$

Finally, by employing the substitution $\varepsilon(\vartheta)=v$ with $\sigma=$ $\mathscr{G}_{1} \circ \mathcal{E}^{-1}, f=g_{2} \circ \mathcal{E}^{-1}$, and $h=\alpha \circ \mathcal{E}^{-1}$, system (9) is exactly system (1).

To go directly to the theme of this paper, we now only review some former results closely related. For the onedimensional version of model (1),

$$
\begin{aligned}
v_{t}-(h(v) P)_{x} & =0, \quad x \in \mathbb{R}, t>0, \\
P_{t}+\sigma(v)_{x} & =f(v) P, \quad x \in \mathbb{R}, t>0,
\end{aligned}
$$

the existence and asymptotic behavior of smooth solutions for the Cauchy problem and initial boundary value problem have been considered by [5, 6] and [7], respectively. In [5], they obtained the convergence rates of the smooth solutions for the Cauchy problem under the following technical assumptions for the nonlinear damping:

$$
\begin{aligned}
f^{\prime \prime}(v) & <0, \\
3\left(f^{\prime}(v)\right)^{2}-f(v) f^{\prime \prime}(v) & <0 .
\end{aligned}
$$

The authors in [6] removed restriction (11). Recently, the authors in [7] proved $L^{p}$ convergence rates for the half space problem. Moreover, the authors in [3] and [4] considered phase transition and the effect of damping respectively. When $f(v)=-b$ with $b$ being a given positive constant and $h(v)=1$, system (10) reduces to the well-known $p$-system with linear damping. The investigation of the case of $p$-system $(h(v)=$ 1) with linear damping $(f(v)=-b)$ has been extensively studied. We refer reader to [8-46] and references therein.

To sum up, it is still unknown on the decay rates of solutions to the $3 \mathrm{D}$ quasilinear hyperbolic system with nonlinear damping (1)-(2). In this paper, we will give a positive answer to this question. More precisely, we prove global existence and uniqueness of strong solutions when the initial data is near its equilibrium in the sense of $H^{3}$-norm. Moreover, if, additionally, $L^{p}$-norm $(1 \leq p<6 / 5)$ of the initial perturbation is finite, we also show the optimal $L^{p}-L^{2}$ decay rates for the solutions without the additional technical assumptions for the nonlinear damping $f(v)$ given by Li and Saxton in [5].

Before stating the main results, we introduce some notations for the use throughout this paper. We use $\|\cdot\|_{m}$ and $\|\cdot\|_{m, r}$ to denote the usual Sobolev spaces with norm $H^{m}\left(\mathbb{R}^{3}\right)$ and $W^{m, r}\left(\mathbb{R}^{3}\right)$, respectively, for $m \geq 0$ and $r \geq 1$. In particular, for $m=0$, we will use $\|\cdot\|$ and $\|\cdot\|_{L^{r}}$ for simplicity. We use $\langle\cdot, \cdot\rangle$ to denote the inner-product in $L^{2}\left(\mathbb{R}^{3}\right)$ and $\Lambda^{s}$ to denote the pseudo differential operator:

$$
\Lambda^{s} z=\mathscr{F}^{-1}\left(|\xi|^{s} \mathscr{F} z\right), \quad \text { for } s \in \mathbb{R}
$$

We will use the notation $a \leq b$ to mean that $a \leq C b$ for a generic constant $C>0$ which may vary at different places.

Now, we are in a position to state our main results.

Theorem 1. Assume that $\sigma \in C^{4}, \sigma^{\prime}<0, h \in C^{4}, h(v)>$ $0, f \in C^{3}, f(v)<0, \bar{v}>0$, and $\left\|\left(v_{0}-\bar{v}, \mathbf{P}_{0}\right)\right\|_{3}$ is small enough. Then the Cauchy problem (1)-(2) has a unique global solution $(v, \mathbf{P})$ with $v>0$, which satisfies

$$
v-\bar{v}, \mathbf{P} \in C^{0}\left(0, \infty ; H^{3}\left(\mathbb{R}^{3}\right)\right) \cap C^{1}\left(0, \infty ; H^{2}\left(\mathbb{R}^{3}\right)\right) .
$$

Furthermore, for any $t \geq 0$, the following energy estimates hold:

$$
\begin{aligned}
& \|(v-\bar{v}, \mathbf{P})(t)\|_{3}^{2}+\int_{0}^{t}\left(\|\nabla v(s)\|_{2}^{2}+\|\mathbf{P}(s)\|_{3}^{2}\right) d s \\
& \quad \leq\left\|\left(v_{0}-\bar{v}, \mathbf{P}_{0}\right)\right\|_{3}^{2} .
\end{aligned}
$$

Finally, if further $\left\|v_{0}-\bar{v}\right\|_{L^{p}}+\left\|\mathbf{P}_{0}\right\|_{L^{3 p /(3-p)}}$ is bounded for some $1 \leq p<6 / 5$, then the following decay estimates of the solution $(v, \mathbf{P})$ hold:

$$
\begin{aligned}
& \|(v-\bar{v})(t)\|_{L^{q}} \lesssim(1+t)^{-(3 / 2)(1 / p-1 / q)}, \quad 2 \leq q \leq 6, \\
& \|(v-\bar{v})(t)\|_{L^{q}} \lesssim(1+t)^{-(3 / 2)(1 / p-1 / 2)-1 / 2},
\end{aligned}
$$$$
6 \leq q \leq \infty,
$$

$$
\|\mathbf{P}(t)\|_{L^{q}} \lesssim(1+t)^{-(3 / 2)(1 / p-1 / 2)-1 / 2},
$$

$$
2 \leq q \leq \infty
$$

$$
\begin{gathered}
\|\nabla(v, \mathbf{P})(t)\|_{2} \lesssim(1+t)^{-(3 / 2)(1 / p-1 / 2)-1 / 2}, \\
\left\|\partial_{t}(v, \mathbf{P})(t)\right\| \lesssim(1+t)^{-(3 / 2)(1 / p-1 / 2)-1 / 2} .
\end{gathered}
$$

We now comment on the proof of Theorem 1. Roughly speaking, we follow the framework of $[47,48]$ on two-phase fluid model, and the proof consists of following three steps.

Firstly, we deduce the optimal $L^{p}-L^{2}$ decay rates on the solutions $(v, \mathbf{P})$ to the corresponding linearized system. By making delicate pointwise analysis on the linearized system, we can prove that the variables $v$ and $\mathbf{P}$ have the $L^{2}$ decay rates $(1+t)^{-(3 / 2)(1 / p-1 / 2)}$ and $(1+t)^{-(3 / 2)(1 / p-1 / 2)-1 / 2}$, respectively, when $\left\|v_{0}-\bar{v}\right\|_{L^{p}}+\left\|\mathbf{P}_{0}\right\|_{L^{3 p /(3-p)}}$ is finite with $1 \leq p \leq 2$. Due to the fact that the Fourier transform of $(30)_{1}-(30)_{2}$ has multiple eigenvalue and the semigroup theory, to investigate spectral structure of $(30)_{1}-(30)_{2}$, we need to analyze the corresponding Jordan structure. Our main idea is to introduce Hodge decomposition to overcome this difficulty. By applying Hodge decomposition to system $(30)_{1}-(30)_{2}$, we can transform system $(30)_{1}-(30)_{2}$ into two systems. One only has one equation, and another has two different eigenvalues.

Secondly, we establish the uniform energy estimates to the original system $(18)_{1}-(18)_{2}$. Based on the system's special 
dissipation structure and delicate analysis and interpolation technique on the nonlinear terms, the desired energy estimates can be achieved. Compared to the one-dimensional results in [5-7], the approach is new and quite different here. In [5-7], the antiderivative technique plays an essential role in proving their main results. However, the antiderivative technique does not work in our high dimensional problem since the antiderivative technique is basically limited to onedimensional problem. The main difficulties in this step of the paper arise from the terms involving $\nabla^{k+1} v$ or $\nabla^{k} \operatorname{div} \mathbf{P}$ which are not included in left hand side of (65) (see Lemma 6 for more details). We use the special dissipation structure of $(18)_{1}-(18)_{2}$, the technique on interpolation and energy estimates, and a technical lemma on estimating the spatial derivatives of nonlinear function to tackle these difficulties. It is worth mentioning that, in our proofs, we do not need the technical assumptions (11) as in [5].

Finally, we deduce the decay rates on the solutions. By virtue of the results obtained in the first two steps and by virtue of the uniform nonlinear energy estimates and the optimal $L^{p}-\mathrm{L}^{2}$ decay rates for the linearized system, we can prove the optimal $L^{p}-L^{2}$ decay rates for the solutions.

The rest of the paper is organized as follows. In Section 2, we first reformulate the problem and then prove Theorem 1 . In Sections 3 and 4 , we derive the optimal $L^{p}-L^{2}$ decay estimates for the linearized system and the uniform nonlinear estimates for the original equations, respectively. In Section 5, we prove Proposition 3 by combining the optimal $L^{p}-L^{2}$ decay estimates obtained in Section 3 and the uniform nonlinear estimates obtained in Section 4.

\section{Reformulation and the Proof of Theorem 1}

Denoting

$$
\begin{aligned}
\lambda & =\sqrt{\frac{-\sigma^{\prime}(\bar{v})}{h(\bar{v})}}, \\
\lambda_{1} & =-\sqrt{-\sigma^{\prime}(\bar{v}) h(\bar{v})}
\end{aligned}
$$

and making change of variables by

$$
(v, \mathbf{P}) \longrightarrow(v+\bar{v}, \lambda \mathbf{P}),
$$

we can reformulate the Cauchy problem (1)-(2) as

$$
\begin{aligned}
v_{t}+\lambda_{1} \operatorname{div} \mathbf{P} & =F, \\
\mathbf{P}_{t}+\lambda_{1} \nabla v-f(\bar{v}) \mathbf{P} & =G, \\
\left.(v, \mathbf{P})\right|_{t=0} & :=\left(v_{0}, \mathbf{P}_{0}\right) \longrightarrow(0,0), \\
\text { as }|x| & \longrightarrow \infty,
\end{aligned}
$$

where

$$
\begin{aligned}
& F(v, \mathbf{P})=(h(v+\bar{v})-h(\bar{v})) \operatorname{div} \mathbf{P}+\mathbf{P} \cdot \nabla h(v), \\
& G(v, \mathbf{P})=(f(v+\bar{v})-f(\bar{v})) \mathbf{P} \\
&+\frac{1}{\lambda}\left(\sigma^{\prime}(\bar{v})-\sigma^{\prime}(v+\bar{v})\right) \nabla v .
\end{aligned}
$$

Here and in the sequel, for the notational simplicity, we will denote the reformulated variables by $(v, \mathbf{P})$.

As usual, Theorem 1 will be proved by combining the local existence result together with uniform a priori energy estimates.

Proposition 2 (local existence). Let $\left(v_{0}, \mathbf{P}_{0}\right) \in H^{3}\left(\mathbb{R}^{3}\right)$ be such that

$$
\inf _{x \in \mathbb{R}^{3}}\left\{v_{0}(x)+\bar{v}\right\}>0 \text {. }
$$

Then there exists a positive constant $T_{0}$ depending on $\left\|\left(v_{0}, \mathbf{P}_{0}\right)\right\|_{3}$ such that the unique solution of the Cauchy problem (18) exists on $\left[0, T_{0}\right]$ and satisfies

$$
v, \mathbf{P} \in C^{0}\left(0, T_{0} ; H^{3}\left(\mathbb{R}^{3}\right)\right) \cap C^{1}\left(0, T_{0} ; H^{2}\left(\mathbb{R}^{3}\right)\right) .
$$

Furthermore, one has the following estimates:

$$
\begin{aligned}
\|(v, \mathbf{P})\|_{3} & \leq 2\left\|\left(v_{0}, \mathbf{P}_{0}\right)\right\|_{3}, \\
\inf _{x \in \mathbb{R}^{3}, 0 \leq t \leq T_{0}}\{v(x, t)+\bar{v}\} & >0 .
\end{aligned}
$$

Proposition 3 (a priori estimate). Let $\left(v_{0}, \mathbf{P}_{0}\right) \in H^{3}\left(\mathbb{R}^{3}\right)$. Assume that the Cauchy problem (18) has a solution $(v, \mathbf{P})(x, t)$ in the same function class as in Proposition 2 on $\mathbb{R}^{3} \times[0, T]$, where $T>0$ is a positive constant. Then there exists a small positive constant $\delta$, which is independent of $T$, such that if

$$
\sup _{0 \leq t \leq T}\|(v, \mathbf{P})(t)\|_{3} \leq \delta,
$$

then, for any $t \in[0, T]$, it holds that

$$
\begin{aligned}
& \|(v, \mathbf{P})(t)\|_{3}^{2}+\int_{0}^{t}\left(\|\nabla v(s)\|_{2}^{2}+\|\mathbf{P}(s)\|_{3}^{2}\right) d s \\
& \quad \leq\left\|\left(v_{0}, \mathbf{P}_{0}\right)\right\|_{3}^{2} .
\end{aligned}
$$

Furthermore, if additionally $\left\|v_{0}-\bar{v}\right\|_{L^{p}}+\left\|\mathbf{P}_{0}\right\|_{L^{3 p /(3-p)}}$ is bounded for some $1 \leq p<6 / 5$, then, for any $t \in[0, T]$, the following decay estimates of the solution $(v, \mathbf{P})$ hold:

$$
\begin{array}{ll}
\|v(t)\|_{L^{q}} \leq(1+t)^{-(3 / 2)(1 / p-1 / q)}, & 2 \leq q \leq 6, \\
\|v(t)\|_{L^{q}} \leq(1+t)^{-(3 / 2)(1 / p-1 / 2)-1 / 2}, & \\
& 6 \leq q \leq \infty, \\
\|\mathbf{P}(t)\|_{L^{q}} \leq(1+t)^{-(3 / 2)(1 / p-1 / 2)-1 / 2}, & \\
& 2 \leq q \leq \infty,
\end{array}
$$

Proof of Theorem 1. Theorem 1 follows from Propositions 2 and 3 by the standard continuity argument. The proof of Proposition 2 is standard whose proof can be found in $[49,50]$. Proposition 3 will be proved in Section 5. 


\section{3. $L^{P}-L^{2}$ Decay Estimates for the Linearized Equations}

The corresponding linearized system to (18) is

$$
\begin{aligned}
& v_{t}+\lambda_{1} \operatorname{div} \mathbf{P}=0, \\
& \mathbf{P}_{t}+\lambda_{1} \nabla v-f(\bar{v}) \mathbf{P}=0, \\
&\left.(v, \mathbf{P})\right|_{t=0}:=\left(v_{0}, \mathbf{P}_{0}\right) \longrightarrow(0,0), \\
& \text { as }|x| \longrightarrow \infty .
\end{aligned}
$$

Due to the fact that the Fourier transform of $(30)_{1}-(30)_{2}$ has multiple eigenvalue and the semigroup theory, to analyze spectral structure of $(30)_{1}-(30)_{2}$, we need to consider corresponding Jordan structure. In spirit of $[40,47,48]$, we will use Hodge decomposition technique to overcome this difficulty. By applying Hodge decomposition to system $(30)_{1}-(30)_{2}$, we can transform system $(30)_{1}-(30)_{2}$ into two systems. One only has one equation, and another has two different eigenvalues. To begin with, let

$$
\phi=\Lambda^{-1} \operatorname{div} \mathbf{P}
$$

be the "compressible part" of $\mathbf{P}$, and let

$$
\psi=\Lambda^{-1} \operatorname{curl} \mathbf{P}
$$

be the "incompressible part" of $\mathbf{P}\left(\right.$ with $\left.(\operatorname{curl} z)_{i}^{j}=\partial_{j} z^{i}-\partial_{i} z^{j}\right)$, then we can rewrite system $(30)_{1}-(30)_{2}$ as follows:

$$
\begin{aligned}
v_{t}+\lambda_{1} \Lambda \phi & =0, \\
\phi_{t}-\lambda_{1} \Lambda v-f(\bar{v}) \phi & =0, \\
\psi_{t}-f(\bar{v}) \psi & =0 .
\end{aligned}
$$

Noticing the definitions of $\phi$ and $\psi$ and the relation

$$
\mathbf{P}=-\Lambda^{-1} \nabla \phi-\Lambda^{-1} \operatorname{div} \psi
$$

which involves pseudo differential operators with zero degree, one can easily see that the estimates in space $H^{3}\left(\mathbb{R}^{3}\right)$ for the velocity $\mathbf{P}$ are the same as for $(\phi, \psi)$.

In the rest of this section, we devote ourselves to show the following $L^{p}-L^{2}$ decay estimates on the linearized system (33).

Proposition 4. Assume that $U_{0}=\left(v_{0}, \mathbf{P}_{0}\right) \in H^{3}\left(\mathbb{R}^{3}\right)$ and $\left\|v_{0}\right\|_{L^{p}}+\left\|\mathbf{P}_{0}\right\|_{L^{3 p /(3-p)}}$ is bounded with $1 \leq p \leq 2$. Denote $U=(\nu, \mathbf{P})$ by the solution of $(30)_{1}-(30)_{2}$, and let $\phi:=\Lambda^{-1} \operatorname{div} \mathbf{P}$ and $\psi=\Lambda^{-1}$ curl $\mathbf{P}$. Then, for $0 \leq k \leq 3$, one has

$$
\begin{aligned}
& \left\|\nabla^{k} \psi(t)\right\| \lesssim e^{f(\bar{v}) t}\left\|\nabla^{k} \psi_{0}\right\|, \\
& \left\|\nabla^{k} v(t)\right\| \lesssim(1+t)^{-(3 / 2)(1 / p-1 / 2)-k / 2} \\
& \cdot\left(\left\|v_{0}\right\|_{L^{p}}+\left\|\mathbf{P}_{0}\right\|_{L^{3 p}(3-p)}+\left\|\nabla^{k} U_{0}\right\|\right), \\
& \left\|\nabla^{k}(\phi, \mathbf{P})(t)\right\| \lesssim(1+t)^{-(3 / 2)(1 / p-1 / 2)-k / 2-1 / 2} \\
& \cdot\left(\left\|v_{0}\right\|_{L^{p}}+\left\|\mathbf{P}_{0}\right\|_{L^{3 p} /(3-p)}+\left\|\nabla^{k} U_{0}\right\|\right) .
\end{aligned}
$$

Proof. Since the proof of (35) is trivial, we will focus on the proofs of (36) and (37). Firstly, in terms of semigroup theory, the solution $(v, \phi)$ of system $(33)_{1}-(33)_{2}$ has the following expression:

$$
(v, \phi)^{t}(t)=G(t) *\left(v_{0}, \phi_{0}\right)^{t}, \quad t \geq 0,
$$

where $G(t):=G(x, t)$ is Green's matrix of system (33) ${ }_{1}$ $(33)_{2}$. Next, we derive the explicit expression for the Fourier transform $\widehat{G}(\xi, t)$ of Green's matrix $G(x, t)$. Taking the Fourier transform to $(33)_{1}-(33)_{2}$, we obtain

$$
\begin{array}{r}
\widehat{v}_{t}+\lambda_{1}|\xi| \widehat{\phi}=0, \\
\widehat{\phi}_{t}-\lambda_{1}|\xi| \widehat{v}-f(\bar{v}) \hat{\phi}=0 .
\end{array}
$$

From (39) and a simple calculation, we have

$$
\begin{aligned}
\widehat{\phi}_{t t}-f(\bar{v}) \widehat{\phi}_{t}+\lambda_{1}^{2}|\xi|^{2} \widehat{\phi} & =0 \\
\widehat{\phi}(\xi, 0) & =\widehat{\phi}_{0} \\
\widehat{\phi}_{t}(\xi, 0) & =\lambda_{1}|\xi| \widehat{v}_{0}+f(\bar{v}) \widehat{\phi}_{0}
\end{aligned}
$$

By a straightforward computation, we can get the expression of the solution to the ODE (40)

$$
\widehat{\phi}(\xi, t)=\frac{\lambda_{1}|\xi|\left(e^{\lambda_{+} t}-e^{\lambda_{-} t}\right)}{\lambda_{+}-\lambda_{-}} \widehat{v}_{0}+\frac{\lambda_{+} e^{\lambda_{+} t}-\lambda_{-} e^{\lambda_{-} t}}{\lambda_{+}-\lambda_{-}} \widehat{\phi}_{0},
$$

where $\lambda_{ \pm}(\xi)=(f(\bar{v}) / 2)\left(1 \pm \sqrt{1-4 \lambda_{1}^{2}|\xi|^{2} /(f(\bar{v}))^{2}}\right)$ are the eigenvalues of the ODE (40). To compute $\widehat{v}$, we substitute (41) into (39) to get

$$
\widehat{v}(\xi, t)=\frac{\lambda_{+} e^{\lambda_{-} t}-\lambda_{-} e^{\lambda_{+} t}}{\lambda_{+}-\lambda_{-}} \widehat{v}_{0}-\frac{\lambda_{1}|\xi|\left(e^{\lambda_{+} t}-e^{\lambda_{-} t}\right)}{\lambda_{+}-\lambda_{-}} \widehat{\phi}_{0}
$$

Thus, from (41)-(42), we obtain the explicit expression of the Fourier transformation $\widehat{G}(\xi, t)$ of Green's matrix $G(x, t)$ as

$$
\begin{aligned}
& \widehat{G}(\xi, t) \\
& \quad=\left(\begin{array}{cc}
\frac{\lambda_{+} e^{\lambda_{-} t}-\lambda_{-} e^{\lambda_{+} t}}{\lambda_{+}-\lambda_{-}} & -\frac{\lambda_{1}|\xi|\left(e^{\lambda_{+} t}-e^{\lambda_{-} t}\right)}{\lambda_{+}-\lambda_{-}} \\
\frac{\lambda_{1}|\xi|\left(e^{\lambda_{+} t}-e^{\lambda_{-}}\right)}{\lambda_{+}-\lambda_{-}} & \frac{\lambda_{+} e^{\lambda_{+} t}-\lambda_{-} e_{-} t}{\lambda_{+}-\lambda_{-}}
\end{array}\right) .
\end{aligned}
$$


In order to deduce the long-time decay estimates of solutions in $L^{p}-L^{2}$-framework, we need to verify the approximation of $\widehat{G}(\xi, t)$. By virtue of the definition $\lambda_{ \pm}(\xi)$, for $|\xi| \ll 1$, it holds that

$$
\begin{aligned}
& \frac{\lambda_{+} e^{\lambda_{-} t}-\lambda_{-} e^{\lambda_{+} t}}{\lambda_{+}-\lambda_{-}} \\
& \sim \frac{f(\bar{v}) e^{\left(\lambda_{1}^{2}|\xi|^{2} / f(\bar{v}) t\right.}-\left(\lambda_{1}^{2}|\xi|^{2} / f(\bar{v})\right) e^{f(\bar{v}) t}}{f(\bar{v})}, \quad|\xi| \ll 1, \\
& \frac{e^{\lambda_{+} t}-e^{\lambda_{-} t}}{\lambda_{+}-\lambda_{-}} \sim \frac{e^{f(\bar{v}) t}-e^{\left(\lambda_{1}^{2}|\xi|^{2} / f(\bar{v})\right) t}}{f(\bar{v})}, \quad|\xi| \ll 1, \\
& \frac{\lambda_{+} e^{\lambda_{+} t}-\lambda_{-} e^{\lambda_{-} t}}{\lambda_{+}-\lambda_{-}} \quad \\
& \sim \frac{f(\bar{v}) e^{f(\bar{v}) t}-\left(\lambda_{1}^{2}|\xi|^{2} / f(\bar{v})\right) e^{\left(\lambda_{1}^{2}|\xi|^{2} / f(\bar{v}) t\right.}}{f(\bar{v})},
\end{aligned}
$$

$$
|\xi| \ll 1 .
$$

Similarly, for high frequency, it also holds that

$$
\begin{aligned}
& \frac{\lambda_{+} e^{\lambda_{-} t}-\lambda_{-} e^{\lambda_{+} t}}{\lambda_{+}-\lambda_{-}} \\
& \sim\left[\cos (\eta t)-\frac{f(\bar{v})}{2} \frac{\sin (\eta t)}{\eta}\right] e^{(f(\bar{v}) / 2) t}, \quad|\xi| \gg R, \\
& \frac{e^{\lambda_{+} t}-e^{\lambda_{-} t}}{\lambda_{+}-\lambda_{-}} \sim \frac{\sin (\eta t)}{\eta} e^{(f(\bar{v}) / 2) t}, \quad|\xi| \gg R, \\
& \frac{\lambda_{+} e^{\lambda_{+} t}-\lambda_{-} e^{\lambda_{-} t}}{\lambda_{+}-\lambda_{-}} \\
& \sim\left[\cos (\eta t)+\frac{f(\bar{v})}{2} \frac{\sin (\eta t)}{\eta}\right] e^{(f(\bar{v}) / 2) t}, \quad|\xi| \gg R,
\end{aligned}
$$

where $R$ is some given positive constant and

$$
\eta=-\frac{f(\bar{v})}{2} \sqrt{\frac{4 \lambda_{1}^{2}|\xi|^{2}}{(f(\bar{v}))^{2}}-1} \sim O(|\xi|), \quad|\xi| \gg R .
$$

By virtue of formula (43) and the asymptotical estimates on its elements, we are in a position to prove (36) and (37). By virtue of (43)-(45), (47)-(48), (50), Parsevel's identity, HausdorffYoung's inequality, and Hölder inequality, we deduce that, for each $0 \leq k \leq 3$,

$$
\begin{aligned}
& \left\|\nabla^{k} v(t)\right\|^{2}=\int_{|\xi| \leq R}|\xi|^{2 k}|\widehat{v}(\xi, t)|^{2} d \xi+\int_{|\xi| \geq R}|\xi|^{2 k} \\
& \cdot|\widehat{v}(\xi, t)|^{2} d \xi \\
& \quad \leq \int_{|\xi| \leq R}\left[|\xi|^{k}\left(e^{\left(\lambda_{1}^{2}|\xi|^{2} / f(\bar{v}) t\right.}+|\xi|^{2} e^{f(\bar{v}) t}\right)\left|\widehat{v}_{0}\right|\right. \\
& \left.+|\xi|^{k+1}\left(e^{\left(\lambda_{1}^{2}|\xi|^{2} / f(\bar{v}) t\right.}+e^{f(\bar{v}) t}\right)\left|\widehat{\phi}_{0}\right|\right]^{2} d \xi \\
& +\int_{|\xi| \geq R} e^{f(\bar{v}) t}|\xi|^{2 k}\left(\left|\widehat{v}_{0}\right|+\left|\widehat{\phi}_{0}\right|\right)^{2} d \xi \leq(1 \\
& +t)^{-(3 / 2)(2 / p-1)-k}\left(\left\|\widehat{v}_{0}\right\|_{L^{p /(p-1)}}^{2}+\left\|\widehat{\phi}_{0}\right\|_{L^{3 p /(4 p-3)}}^{2}\right) \\
& +e^{f(\bar{v}) t}\left\|\left(|\xi|^{k} \widehat{v}_{0},|\xi|^{k} \widehat{\phi}_{0}\right)\right\|^{2} \leq(1+t)^{-(3 / 2)(2 / p-1)-k} \\
& \quad \cdot\left(\left\|v_{0}\right\|_{L^{p}}^{2}+\left\|\mathbf{P}_{0}\right\|_{L^{3 p /(3-p)}}^{2}+\left\|\nabla^{k} U_{0}\right\|^{2}\right) .
\end{aligned}
$$

This proves (36). Similarly, for each $0 \leq k \leq 3$, we also have

$$
\left\|\nabla^{k} \phi(t)\right\|^{2}=\int_{|\xi| \leq R}|\xi|^{2 k}|\widehat{\phi}(\xi, t)|^{2} d \xi+\int_{|\xi| \geq R}|\xi|^{2 k}
$$

$$
\begin{aligned}
& \cdot|\widehat{\phi}(\xi, t)|^{2} d \xi \\
& \leq \int_{|\xi| \leq R}\left[|\xi|^{k+1}\left(e^{\left(\lambda_{1}^{2}|\xi|^{2} / f(\bar{v}) t\right.}+e^{f(\bar{v}) t}\right)\left|\widehat{\phi}_{0}\right|\right. \\
& \left.+|\xi|^{k}\left(|\xi|^{2} e^{\left(\lambda_{1}^{2}|\xi|^{2} / f(\bar{v})\right) t}+e^{f(\bar{v}) t}\right)\left|\widehat{v}_{0}\right|\right]^{2} d \xi \\
& +\int_{|\xi| \geq R} e^{f(\bar{v}) t}|\xi|^{2 k}\left(\left|\widehat{v}_{0}\right|+\left|\widehat{\phi}_{0}\right|\right)^{2} d \xi \lesssim(1 \\
& +t)^{-(3 / 2)(2 / p-1)-k-1}\left(\left\|\widehat{v}_{0}\right\|_{L^{p /(p-1)}}^{2}+\left\|\widehat{\phi}_{0}\right\|_{L^{3 p /(4 p-3)}}^{2}\right) \\
& +e^{f(\bar{v}) t}\left\|\left(|\xi|^{k} \widehat{v}_{0},|\xi|^{k} \widehat{\phi}_{0}\right)\right\|^{2} \leq(1 \\
& +t)^{-(3 / 2)(2 / p-1)-k-1}\left(\left\|v_{0}\right\|_{L^{p}}^{2}+\left\|\mathbf{P}_{0}\right\|_{L^{3 p /(3-p)}}^{2}\right. \\
& \left.+\left\|\nabla^{k} U_{0}\right\|^{2}\right),
\end{aligned}
$$

which together with (34)-(35) yields (37).

Therefore, we have proved Proposition 4.

\section{Uniform a Priori Estimates}

In this section, we deduce the uniform a priori estimates stated in Proposition 3. Throughout this section, we assume that all the conditions in Proposition 3 are satisfied. Furthermore, we assume a priori that for sufficiently small $\delta>0$

$$
\sup _{0 \leq t \leq T}\|(v, \mathbf{P})(t)\|_{3} \leq \delta .
$$


We first derive the following lower order energy estimate of the solutions.

Lemma 5. There exists a suitably large constant $D_{1}>0$, which is independent of $\delta$, such that

$$
\begin{aligned}
\frac{d}{d t}\left(D_{1}\|(v, \mathbf{P})(t)\|^{2}+\langle\nabla v, \mathbf{P}\rangle(t)\right) \\
\quad+C\left(\|\nabla v(t)\|^{2}+\|\mathbf{P}(t)\|^{2}\right) \lesssim\|\nabla \mathbf{P}(t)\|^{2},
\end{aligned}
$$

for any $0 \leq t \leq T$.

Proof. Multiplying $(18)_{1}-(18)_{2}$ by $v, \mathbf{P}$, respectively, and then integrating them over $\mathbb{R}^{3}$, we have

$$
\frac{1}{2} \frac{d}{d t}\|(v, \mathbf{P})\|^{2}-f(\bar{v})\|\mathbf{P}\|^{2}=\langle v, F\rangle+\langle\mathbf{P}, G\rangle .
$$

We will estimate the two terms in the right-hand side of (55) as follows.

First, for the first term, by virtue of Hölder inequality, Cauchy inequality, and Lemmas A.1 and A.4, we get

$$
\begin{aligned}
|\langle v, F\rangle| \lesssim & |\langle v,(h(v+\bar{v})-h(\bar{v}) \operatorname{div} \mathbf{P})\rangle| \\
& +|\langle v, \mathbf{P} \cdot \nabla h(v)\rangle| \\
\lesssim & \|v\|_{L^{6}}\|h(v+\bar{v})-h(\bar{v})\|_{L^{3}}\|\nabla \mathbf{P}\| \\
& +\|v\|_{L^{3}}\|\nabla h(v)\|\|\mathbf{P}\|_{L^{6}} \\
\lesssim & \|\nabla v\|\|v\|_{H^{1}}\|\nabla \mathbf{P}\|+\|v\|_{H^{1}}\|\nabla v\|\|\nabla \mathbf{P}\| \\
\lesssim & \delta\left(\|\nabla v\|^{2}+\|\nabla \mathbf{P}\|^{2}\right) .
\end{aligned}
$$

Similarly, for the second term, it also holds that

$$
\begin{aligned}
|\langle\mathbf{P}, G\rangle| \lesssim & |\langle\mathbf{P},(f(v+\bar{v})-f(\bar{v})) \mathbf{P}\rangle| \\
& +\left|\left\langle\mathbf{P},\left(\sigma^{\prime}(\bar{v})-\sigma^{\prime}(v+\bar{v})\right) \nabla v\right\rangle\right| \\
\lesssim & \|f(v+\bar{v})-f(\bar{v})\|_{L^{\infty}}\|\mathbf{P}\|^{2} \\
& +\|\mathbf{P}\|_{L^{6}}\|\nabla v\|\left\|\sigma^{\prime}(\bar{v})-\sigma^{\prime}(v+\bar{v})\right\|_{L^{3}} \\
\lesssim & \|v\|_{L^{\infty}}\|\mathbf{P}\|^{2}+\|\nabla \mathbf{P}\|\|\nabla v\|\|v\|_{H^{1}} \\
\lesssim & \delta\left(\|\mathbf{P}\|^{2}+\|\nabla \mathbf{P}\|^{2}+\|\nabla v\|^{2}\right) .
\end{aligned}
$$

Thus substituting (56) and (57) into (55) gives

$$
\frac{d}{d t}\|(v, \mathbf{P})\|^{2}+C\|\mathbf{P}\|^{2} \lesssim \delta\left(\|\nabla v\|^{2}+\|\nabla \mathbf{P}\|^{2}\right),
$$

since $f(\bar{v})<0$ and $\delta>0$ is suitably small.

Next we shall estimate the term $\|\nabla v\|^{2}$. Multiplying $(18)_{2}$ by $\nabla v$ and then integrating it over, we obtain

$$
\lambda_{1}\|\nabla v\|^{2}=\left\langle-\mathbf{P}_{t}, \nabla v\right\rangle+\langle f(\bar{v}) \mathbf{P}, \nabla v\rangle+\langle G, \nabla v\rangle,
$$

where from $(18)_{1}$, we can rewrite the first term in the righthand side as

$$
\begin{aligned}
\left\langle-\mathbf{P}_{t}, \nabla v\right\rangle & =-\frac{d}{d t}\langle\nabla v, \mathbf{P}\rangle+\left\langle\nabla v_{t}, \mathbf{P}\right\rangle \\
& =-\frac{d}{d t}\langle\nabla v, \mathbf{P}\rangle-\left\langle v_{t}, \operatorname{div} \mathbf{P}\right\rangle \\
& =-\frac{d}{d t}\langle\nabla v, \mathbf{P}\rangle+\left\langle\lambda_{1} \operatorname{div} \mathbf{P}-F, \operatorname{div} \mathbf{P}\right\rangle .
\end{aligned}
$$

By virtue of the definition of $F$, we obtain

$$
\begin{aligned}
& |\langle-F, \operatorname{div} \mathbf{P}\rangle| \lesssim\left(\|h(v+\bar{v})-h(\bar{v})\|_{L^{\infty}}\|\nabla \mathbf{P}\|^{2}\right. \\
& \left.+\|\mathbf{P}\|_{L^{6}}\|\nabla \mathbf{P}\|\|\nabla h(v)\|_{L^{3}}\right) \lesssim\left(\|v\|_{L^{\infty}}+\|\nabla v\|_{H^{1}}\right) \\
& \cdot\|\nabla \mathbf{P}\|^{2} \lesssim \delta\|\nabla \mathbf{P}\|^{2},
\end{aligned}
$$

where Lemma A.4 has been used. Then, by combining the relations (59)-(61), we have

$$
\begin{aligned}
\lambda_{1} & \|\nabla v\|^{2}+\frac{d}{d t}\langle\nabla v, \mathbf{P}\rangle \\
= & \langle f(\bar{v}) \mathbf{P}, \nabla v\rangle+\left\langle\lambda_{1} \operatorname{div} \mathbf{P}-F, \operatorname{div} \mathbf{P}\right\rangle+\langle G, \nabla v\rangle \\
& \leqslant\|\mathbf{P}\|^{2}+\frac{\lambda_{1}}{4}\|\nabla v\|^{2}+\lambda_{1}\|\operatorname{div} \mathbf{P}\|^{2}+\delta\|\nabla \mathbf{P}\|^{2} \\
& +\langle G, \nabla v\rangle .
\end{aligned}
$$

Similar to the proof of the estimate on $\langle\mathbf{P}, G\rangle$, it also holds that

$$
|\langle G, \nabla v\rangle| \lesssim \delta\|\nabla v\|^{2} .
$$

Putting (63) into (62) and noting that $\delta>0$ is sufficiently small, we obtain

$$
\frac{d}{d t}\langle\nabla v, \mathbf{P}\rangle+\frac{\lambda_{1}}{2}\|\nabla v\|^{2} \lesssim\|\mathbf{P}\|_{1}^{2} .
$$

Finally, multiplying (58) by a sufficiently large positive constant $D_{1}$ and then adding it to (64), we can get (54) since $\delta>0$ is small enough. Therefore, we have completed the proof of Lemma 5.

In the following lemma, we deduce the higher order energy estimate of the solutions.

Lemma 6. There exists a suitably large constant $D_{2}>0$, which is independent of $\delta$, such that

$$
\begin{aligned}
& \frac{d}{d t}\left(D _ { 2 } \left(\|\nabla(v, \mathbf{P})\|_{2}^{2}\right.\right. \\
& \left.\quad+\sum_{1 \leq k \leq 3} \int_{\mathbb{R}^{3}} \frac{\chi}{\lambda_{1}-\chi}\left|\nabla^{k} v\right|^{2}+\frac{1}{\lambda}\left(\sigma^{\prime}(v+\bar{v})-\sigma^{\prime}(\bar{v})\right)\left|\nabla^{k} \mathbf{P}\right|^{2} d x\right) \\
& \left.\quad+\sum_{1 \leq k \leq 2}\left\langle\nabla \nabla^{k} v, \nabla^{k} \mathbf{P}\right\rangle(t)\right)+C\left(\left\|\nabla^{2} v(t)\right\|_{1}^{2}+\|\nabla \mathbf{P}(t)\|_{2}^{2}\right) \\
& \quad \leq \delta\|\nabla v(t)\|^{2},
\end{aligned}
$$

for any $0 \leq t \leq T$, where $\chi=h(v+\bar{v})-h(\bar{v})$. 
Proof. For $1 \leq k \leq 3$, by applying $\nabla^{k}$ to $(18)_{1}-(18)_{2}$ and multiplying the resulting identities by $\nabla^{k} v, \nabla^{k} \mathbf{P}$, respectively, summing up them, and then integrating over $\mathbb{R}^{3}$, we have

$$
\begin{aligned}
\frac{1}{2} & \frac{d}{d t}\left\|\nabla^{k}(v, \mathbf{P})\right\|^{2}-f(\bar{v})\left\|\nabla^{k} \mathbf{P}\right\|^{2} \\
& =\left\langle\nabla^{k} v, \nabla^{k} F\right\rangle+\left\langle\nabla^{k} \mathbf{P}, \nabla^{k} G\right\rangle:=I_{1}+I_{2} .
\end{aligned}
$$

Next, we will estimate the two terms in the right-hand side of (66). To do this, by the definition of $F$, we get

$$
\begin{aligned}
I_{1}= & \left\langle\nabla^{k} v, \nabla^{k}[(h(v+\bar{v})-h(\bar{v})) \operatorname{div} \mathbf{P}]\right\rangle \\
& +\left\langle\nabla^{k} v, \nabla^{k}(\mathbf{P} \cdot \nabla h(v))\right\rangle \\
:= & \left\langle\nabla^{k} v, \nabla^{k}(\chi(v) \operatorname{div} \mathbf{P})\right\rangle+\left\langle\nabla^{k} v, \nabla^{k}(\mathbf{P} \cdot \nabla h(v))\right\rangle \\
= & \left\langle\nabla^{k} v, \chi(v) \nabla^{k} \operatorname{div} \mathbf{P}\right\rangle+\left\langle\nabla^{k} v,\left[\nabla^{k}, \chi(v)\right] \operatorname{div} \mathbf{P}\right\rangle \\
& +\left\langle\nabla^{k} v, \mathbf{P} \cdot \nabla \nabla^{k} h(v)\right\rangle+\left\langle\nabla^{k} v,\left[\nabla^{k}, \mathbf{P}\right] \nabla h(v)\right\rangle \\
:= & I_{1}^{1}+I_{1}^{2}+I_{1}^{3}+I_{1}^{4} .
\end{aligned}
$$

We will estimate the four terms $I_{1}^{i}$ with $1 \leq i \leq 4$. The main difficulties arise from terms involving $\nabla^{k+1} v$ or $\nabla^{k} \operatorname{div} \mathbf{P}$ which are not included in left hand side of (65). The main observation is that we can tackle these difficulties by using the special dissipation structure of $(18)_{1}-(18)_{2}$, the technique on interpolation and energy estimates, and a technical lemma on estimating the spatial derivatives of nonlinear function to tackle these difficulties. It should be mentioned that, in our proofs, we do not need the technical assumptions (11) as in [5]. Firstly, by virtue of $(18)_{1}, I_{1}^{1}$ can be rewritten as

$$
\begin{aligned}
I_{1}^{1}= & \left\langle-\chi(v) \nabla^{k} v, \nabla^{k}\left(\frac{v_{t}-\mathbf{P} \cdot \nabla h(v)}{\lambda_{1}-\chi(v)}\right)\right\rangle \\
= & \left\langle-\chi(v) \nabla^{k} v, \nabla^{k}\left(\frac{v_{t}}{\lambda_{1}-\chi(v)}\right)\right\rangle \\
& +\left\langle\chi(v) \nabla^{k} v, \nabla^{k}\left(\frac{\mathbf{P} \cdot \nabla h(v)}{\lambda_{1}-\chi(v)}\right)\right\rangle \\
= & \left\langle-\frac{\chi(v)}{\lambda_{1}-\chi(v)} \nabla^{k} v, \nabla^{k} v_{t}\right\rangle \\
& +\left\langle-\chi(v) \nabla^{k} v,\left[\nabla^{k}, \frac{1}{\lambda_{1}-\chi(v)}\right] v_{t}\right\rangle \\
& +\left\langle\frac{\chi(v) \nabla^{k} v}{\lambda_{1}-\chi(v)}, \mathbf{P} \cdot \nabla \nabla^{k} h(v)\right\rangle \\
& +\left\langle\chi(v) \nabla^{k} v,\left[\nabla^{k}, \frac{\mathbf{P}}{\lambda_{1}-\chi(v)}\right] \nabla h(v)\right\rangle \\
= & I_{1}^{1,1}+I_{1}^{1,2}+I_{1}^{1,3}+I_{1}^{1,4} .
\end{aligned}
$$

From (18) ${ }_{1}$ and (53), we obtain

$$
\begin{aligned}
I_{1}^{1,1}= & \frac{1}{2} \frac{d}{d t} \int_{\mathbb{R}^{3}} \frac{-\chi}{\lambda_{1}-\chi}\left|\nabla^{k} v\right|^{2} d x \\
& +\frac{1}{2}\left\langle\left(\frac{\chi}{\lambda_{1}-\chi}\right)_{t},\left|\nabla^{k} v\right|^{2}\right\rangle \\
\lesssim & \frac{1}{2} \frac{d}{d t} \int_{\mathbb{R}^{3}} \frac{-\chi}{\lambda_{1}-\chi}\left|\nabla^{k} v\right|^{2} d x \\
& +\delta \int_{\mathbb{R}^{3}}\left|v_{t}\right|\left|\nabla^{k} v\right|^{2} d x \\
\lesssim & \frac{1}{2} \frac{d}{d t} \int_{\mathbb{R}^{3}} \frac{-\chi}{\lambda_{1}-\chi}\left|\nabla^{k} v\right|^{2} d x+\delta\left\|\nabla^{k} v\right\|^{2} .
\end{aligned}
$$

By virtue of (18) $)_{1}$, (53), Lemmas A.1-A.4, Cauchy inequality, and Hölder inequality, we get

$$
\begin{aligned}
I_{1}^{1,2} & \leq\|\chi\|_{L^{\infty}}\left\|\nabla^{k} v\right\|\left(\left\|\nabla^{k-1} v_{t}\right\|\left\|\nabla\left(\frac{1}{\lambda_{1}-\chi}\right)\right\|_{L^{\infty}}\right. \\
+ & \left.\left\|\nabla^{k}\left(\frac{1}{\lambda_{1}-\chi}\right)\right\|\left\|v_{t}\right\|_{L^{\infty}}\right) \lesssim\|v\|_{L^{\infty}}\left\|\nabla^{k} v\right\| \\
\cdot & \left(\left\|\nabla^{k-1} v_{t}\right\|\|\nabla v\|_{L^{\infty}}+\left\|\nabla^{k} v\right\|\left\|v_{t}\right\|_{L^{\infty}}\right) \leq \delta\left\|\nabla^{k} v\right\| \\
\cdot & \left(\left\|\nabla^{k} \mathbf{P}\right\|+\left\|\nabla^{k-1}(\mathbf{P} \cdot \nabla h(v))\right\|+\left\|\nabla^{k} v\right\|\right) \lesssim \delta\left\|\nabla^{k} v\right\| \\
& \cdot\left(\left\|\nabla^{k} \mathbf{P}\right\|+\|\mathbf{P}\|_{L^{\infty}}\left\|\nabla^{k} h(v)\right\|\right. \\
& \left.+\left\|\nabla^{k-1} \mathbf{P}\right\|_{L^{6}}\|\nabla h(v)\|_{L^{3}}+\left\|\nabla^{k} v\right\|\right) \lesssim \delta\left(\left\|\nabla^{k} v\right\|^{2}\right. \\
& \left.+\left\|\nabla^{k} \mathbf{P}\right\|^{2}\right) .
\end{aligned}
$$

Next, we estimate the term $I_{1}^{1,3}$. If $k=1$, from integration by parts, Lemmas A.1 and A.4, we obtain

$$
\begin{aligned}
I_{1}^{1,3}= & \left\langle\frac{\chi(v) \nabla v}{\lambda_{1}-\chi(v)}, \mathbf{P} \cdot \nabla \nabla h(v)\right\rangle \\
= & \frac{1}{2}\left\langle\frac{\chi(v) h^{\prime}(v)}{\lambda_{1}-\chi(v)} \mathbf{P}, \nabla\left(|\nabla v|^{2}\right)\right\rangle \\
& +\left\langle\frac{\chi(v) h^{\prime \prime}(v) \mathbf{P} \cdot \nabla v}{\lambda_{1}-\chi(v)},|\nabla v|^{2}\right\rangle \\
= & -\frac{1}{2}\left\langle\operatorname{div}\left(\frac{\chi(v) h^{\prime}(v)}{\lambda_{1}-\chi(v)} \mathbf{P}\right),|\nabla v|^{2}\right\rangle \\
& +\left\langle\frac{\chi(v) h^{\prime \prime}(v) \mathbf{P} \cdot \nabla v}{\lambda_{1}-\chi(v)},|\nabla v|^{2}\right\rangle \\
\lesssim & \left(\|v\|_{L^{\infty}}\|\nabla \mathbf{P}\|_{L^{\infty}}+\|\mathbf{P}\|_{L^{\infty}}\|\nabla v\|_{L^{\infty}}\right)\|\nabla v\|^{2} \\
\lesssim & \delta\|\nabla v\|^{2} .
\end{aligned}
$$


If $k=2$, by integration by parts, Hölder inequality, and Lemmas A.1 and A.4, we have

$$
\begin{aligned}
I_{1}^{1,3} & =\left\langle\frac{\chi(v) \nabla^{2} v}{\lambda_{1}-\chi(v)}, \mathbf{P} \cdot \nabla \nabla^{2} h(v)\right\rangle \\
& =\frac{1}{2}\left\langle\frac{\chi(v) h^{\prime}(v)}{\lambda_{1}-\chi(v)} \mathbf{P}, \nabla\left(\left|\nabla^{2} v\right|^{2}\right)\right\rangle \\
& +\left\langle\frac{\chi(v) \nabla^{2} v}{\lambda_{1}-\chi(v)}, \mathbf{P}\right. \\
& \left.\cdot\left(3 h^{\prime \prime}(v) \nabla v \cdot \nabla^{2} v+h^{\prime \prime \prime}(v)|\nabla v|^{2} \nabla v\right)\right\rangle \\
& =-\frac{1}{2}\left\langle\operatorname{div}\left(\frac{\chi(v) h^{\prime}(v)}{\lambda_{1}-\chi(v)} \mathbf{P}\right),\left|\nabla^{2} v\right|^{2}\right\rangle \\
& +\left\langle\frac{\chi(v) \nabla^{2} v}{\lambda_{1}-\chi(v)}, \mathbf{P}\right. \\
& \left.\cdot\left(3 h^{\prime \prime}(v) \nabla v \cdot \nabla^{2} v+h^{\prime \prime \prime}(v)|\nabla v|^{2} \nabla v\right)\right\rangle \\
& \leqslant \delta\left\|\nabla^{2} v\right\|^{2}+\|\mathbf{P}\|_{L^{\infty}}\|\nabla v\|_{L^{\infty}}\left\|\nabla^{2} v\right\|^{2}+\|\mathbf{P}\|_{L^{3}}\|\nabla v\|_{L^{6}} \\
& \cdot\|\nabla v\|_{L^{\infty}}^{2}\left\|\nabla^{2} v\right\| \leqslant \delta\left\|\nabla^{2} v\right\|^{2} .
\end{aligned}
$$

If $k=3$, by using similar arguments as the above, we obtain

$$
\begin{aligned}
I_{1}^{1,3} & =\left\langle\frac{\chi(v) \nabla^{3} v}{\lambda_{1}-\chi(v)}, \mathbf{P} \cdot \nabla \nabla^{3} h(v)\right\rangle \\
& \leqslant-\frac{1}{2}\left\langle\operatorname{div}\left(\frac{\chi(v) h^{\prime}(v)}{\lambda_{1}-\chi(v)} \mathbf{P}\right),\left|\nabla^{3} v\right|^{2}\right\rangle \\
& +\int_{\mathbb{R}^{3}}\left(\left|\nabla^{2} v\right|^{2}+\left|\nabla^{3} v\right||\nabla v|+|\nabla v|^{4}\right)\left|\nabla^{3} v\right||\mathbf{P}| d x \\
& \leq \delta\left\|\nabla^{3} v\right\|^{2}+\|\mathbf{P}\|_{L^{\infty}}\left\|\nabla^{3} v\right\|\left(\left\|\nabla^{2} v\right\|_{L^{3}}\left\|\nabla^{2} v\right\|_{L^{6}}\right. \\
& \left.+\|\nabla v\|_{L^{\infty}}\left\|\nabla^{3} v\right\|+\|\nabla v\|_{L^{\infty}}\|v\|_{L^{\infty}}\left\|\nabla^{3} v\right\|\right) \\
& \leq \delta\left\|\nabla^{3} v\right\|^{2} .
\end{aligned}
$$

Combining (71)-(73) gives that

$$
I_{1}^{1,3} \lesssim \delta\left\|\nabla^{k} v\right\|^{2}
$$

From (53), (56), Lemmas A.1-A.4, Cauchy inequality, and Hölder inequality, we get

$$
I_{1}^{1,4} \lesssim\left\|\nabla^{k} v\right\|\left(\left\|\nabla^{k} h(v)\right\|\left\|\frac{\mathbf{P}}{\lambda_{1}-\chi}\right\|_{L^{\infty}}\right.
$$

$$
\begin{aligned}
& \left.+\left\|\nabla^{k}\left(\frac{\mathbf{P}}{\lambda_{1}-\chi}\right)\right\|\|\nabla h(v)\|_{L^{\infty}}\right) \lesssim \delta\left\|\nabla^{k} v\right\| \\
& \cdot\left(\|\mathbf{P}\|_{L^{\infty}}\left\|\nabla^{k} v\right\|+\|\nabla v\|_{L^{\infty}}\left\|\nabla^{k}(v, \mathbf{P})\right\|\right) \lesssim \delta\left(\left\|\nabla^{k} v\right\|^{2}\right. \\
& \left.+\left\|\nabla^{k} \mathbf{P}\right\|^{2}\right) .
\end{aligned}
$$

Putting (69)-(70) and (74)-(75) into (68) leads to

$$
\begin{aligned}
I_{1}^{1} \lesssim & \frac{1}{2} \frac{d}{d t} \int_{\mathbb{R}^{3}} \frac{-\chi}{\lambda_{1}-\chi}\left|\nabla^{k} v\right|^{2} d x \\
& +\delta\left(\left\|\nabla^{k} v\right\|^{2}+\left\|\nabla^{k} \mathbf{P}\right\|^{2}\right) .
\end{aligned}
$$

Similarly, for the terms $I_{1}^{2}$ and $I_{1}^{4}$, we also have

$$
\begin{aligned}
I_{1}^{2} & \leq\left\|\nabla^{k} v\right\| \\
& \cdot\left(\left\|\nabla^{k-1} \operatorname{div} \mathbf{P}\right\|\|\nabla \chi(v)\|_{L^{\infty}}+\left\|\nabla^{k} \chi(v)\right\|\|\operatorname{div} \mathbf{P}\|_{L^{\infty}}\right) \\
& \leq \delta\left\|\nabla^{k} v\right\|\left(\|\nabla v\|_{L^{\infty}}\left\|\nabla^{k} v\right\|+\|\nabla \mathbf{P}\|_{L^{\infty}}\left\|\nabla^{k} v\right\|\right) \\
& \leq \delta\left(\left\|\nabla^{k} v\right\|^{2}+\left\|\nabla^{k} \mathbf{P}\right\|^{2}\right) \\
I_{1}^{4} & \leq\left\|\nabla^{k} v\right\|\left(\left\|\nabla^{k} \mathbf{P}\right\|\|\nabla v\|_{L^{\infty}}+\left\|\nabla^{k} v\right\|\|\nabla \mathbf{P}\|_{L^{\infty}}\right) \\
& \leq \delta\left\|\nabla^{k} v\right\|\left(\left\|\nabla^{k} \mathbf{P}\right\|+\left\|\nabla^{k} v\right\|\right) \\
& \leq \delta\left(\left\|\nabla^{k} v\right\|^{2}+\left\|\nabla^{k} \mathbf{P}\right\|^{2}\right) .
\end{aligned}
$$

Using the similar arguments in obtaining (74), for the term $I_{1}^{3}$, it also holds that

$$
I_{1}^{3} \lesssim \delta\left\|\nabla^{k} v\right\|^{2} .
$$

Putting (76)-(78) into (67) leads to

$$
\begin{aligned}
I_{1} \lesssim & \frac{1}{2} \frac{d}{d t} \int_{\mathbb{R}^{3}} \frac{-\chi}{\lambda_{1}-\chi}\left|\nabla^{k} v\right|^{2} d x \\
& +\delta\left(\left\|\nabla^{k} v\right\|^{2}+\left\|\nabla^{2} \mathbf{P}\right\|^{2}\right) .
\end{aligned}
$$

Next, we estimate the term $I_{2}$. By virtue of the definition of $G$, we separate $I_{2}$ into three parts

$$
\begin{aligned}
I_{2}= & \left\langle\nabla^{k} \mathbf{P}, \nabla^{k}[(f(v+\bar{v})-f(\bar{v})) \mathbf{P}]\right\rangle \\
& +\frac{1}{\lambda}\left\langle\left(\sigma^{\prime}(\bar{v})-\sigma^{\prime}(v+\bar{v})\right) \nabla^{k} \mathbf{P}, \nabla^{k} \nabla v\right\rangle \\
& +\frac{1}{\lambda}\left\langle\nabla^{k} \mathbf{P},\left[\nabla^{k}, \sigma^{\prime}(\bar{v})-\sigma^{\prime}(v+\bar{v})\right] \nabla v\right\rangle \\
:= & I_{2}^{1}+I_{2}^{2}+I_{2}^{3} .
\end{aligned}
$$


From (53), (56), Lemmas A.1-A.4, Cauchy inequality, and Hölder inequality, we get

$$
\begin{aligned}
I_{2}^{1} & \lesssim\left\|\nabla^{k} \mathbf{P}\right\|\left(\|\mathbf{P}\|_{L^{\infty}}\left\|\nabla^{k} v\right\|+\|v\|_{L^{\infty}}\left\|\nabla^{k} \mathbf{P}\right\|\right) \\
& \lesssim \delta\left(\left\|\nabla^{k} v\right\|^{2}+\left\|\nabla^{k} \mathbf{P}\right\|^{2}\right), \\
I_{2}^{3} & \lesssim\|\nabla v\|_{L^{\infty}}\left\|\nabla^{k} v\right\|\left\|\nabla^{k} \mathbf{P}\right\| \lesssim \delta\left(\left\|\nabla^{k} v\right\|^{2}+\left\|\nabla^{k} \mathbf{P}\right\|^{2}\right) .
\end{aligned}
$$

Using similar arguments in obtaining (76), for $I_{2}^{2}$, it also holds that

$$
\begin{aligned}
I_{2}^{3} \lesssim & -\frac{1}{2 \lambda} \frac{d}{d t} \int_{\mathbb{R}^{3}}\left(\sigma^{\prime}(v+\bar{v})-\sigma^{\prime}(\bar{v})\right)\left|\nabla^{k} \mathbf{P}\right|^{2} d x \\
& +\delta\left(\left\|\nabla^{k} v\right\|^{2}+\left\|\nabla^{k} \mathbf{P}\right\|^{2}\right) .
\end{aligned}
$$

Putting the estimates (81)-(82) into (80) gives

$$
\begin{aligned}
I_{2} \lesssim & -\frac{1}{2 \lambda} \frac{d}{d t} \int_{\mathbb{R}^{3}}\left(\sigma^{\prime}(v+\bar{v})-\sigma^{\prime}(\bar{v})\right)\left|\nabla^{k} \mathbf{P}\right|^{2} d x \\
& +\delta\left(\left\|\nabla^{k} v\right\|^{2}+\left\|\nabla^{k} \mathbf{P}\right\|^{2}\right) .
\end{aligned}
$$

Substituting (79) and (83) into (66) and then summing up the resultant equation for $k=1$ to 3 , we obtain

$$
\begin{aligned}
& \frac{d}{d t}\left(\|\nabla(v, \mathbf{P})\|_{2}^{2}+\sum_{1 \leq k \leq 3} \int_{\mathbb{R}^{3}} \frac{\chi}{\lambda_{1}-\chi}\left|\nabla^{k} v\right|^{2}\right. \\
& \left.\quad+\frac{1}{\lambda}\left(\sigma^{\prime}(v+\bar{v})-\sigma^{\prime}(\bar{v})\right)\left|\nabla^{k} \mathbf{P}\right|^{2} d x\right)+\|\nabla \mathbf{P}\|_{2}^{2} \\
& \quad \leq \delta\|\nabla v\|_{2}^{2} .
\end{aligned}
$$

Finally, we derive the estimate on $\left\|\nabla \nabla^{k} v\right\|^{2}$ for $1 \leq k \leq 2$. Applying $\nabla^{k} v$ to $(18)_{2}$, multiplying the resulting identity by $\nabla \nabla^{k} v$, and then integrating over $\mathbb{R}^{3}$, we obtain

$$
\begin{aligned}
\lambda_{1}\left\|\nabla \nabla^{k} v\right\|^{2}= & \left\langle-\nabla \nabla^{k} v, \nabla^{k} \mathbf{P}_{t}\right\rangle+\left\langle\nabla \nabla^{k} v, f(\bar{v}) \nabla^{k} \mathbf{P}\right\rangle \\
& +\left\langle\nabla \nabla^{k} v, \nabla^{k} G\right\rangle,
\end{aligned}
$$

where from $(18)_{1}$, we can rewrite the first term in the righthand side of (85) as

$$
\begin{aligned}
\left\langle-\nabla \nabla^{k} v, \nabla^{k} \mathbf{P}_{t}\right\rangle= & -\frac{d}{d t}\left\langle\nabla \nabla^{k} v, \nabla^{k} \mathbf{P}\right\rangle \\
& +\left\langle\nabla \nabla^{k} v_{t}, \nabla^{k} \mathbf{P}\right\rangle \\
= & -\frac{d}{d t}\left\langle\nabla \nabla^{k} v, \nabla^{k} \mathbf{P}\right\rangle \\
& -\left\langle\nabla^{k} v_{t}, \nabla^{k} \operatorname{div} \mathbf{P}\right\rangle \\
= & -\frac{d}{d t}\left\langle\nabla \nabla^{k} v, \nabla^{k} \mathbf{P}\right\rangle \\
& +\left\langle\lambda_{1} \nabla^{k} \operatorname{div} \mathbf{P}-\nabla^{k} F, \nabla^{k} \operatorname{div} \mathbf{P}\right\rangle .
\end{aligned}
$$

By virtue of the definition of $F,(53),(56)$, and Lemmas A.1-A.4, we get

$$
\begin{aligned}
& \left\langle-\nabla^{k} F, \nabla^{k} \operatorname{div} \mathbf{P}\right\rangle \lesssim\left\|\nabla^{k} \operatorname{div} \mathbf{P}\right\|\left(\left\|\nabla^{k} \operatorname{div} \mathbf{P}\right\|\|\chi\|_{L^{\infty}}\right. \\
& +\left\|\nabla^{k} \chi\right\|\|\operatorname{div} \mathbf{P}\|_{L^{\infty}}+\left\|\nabla^{k+1} v\right\|\|\mathbf{P}\|_{L^{\infty}} \\
& \left.+\left\|\nabla^{k} \mathbf{P}\right\|_{L^{6}}\|\nabla v\|_{L^{3}}\right) \lesssim \delta\left\|\nabla^{k+1} \mathbf{P}\right\|\left(\left\|\nabla^{k+1} \mathbf{P}\right\|\right. \\
& \left.+\left\|\nabla^{k+1} v\right\|\right) \lesssim \delta\left(\left\|\nabla^{k+1} v\right\|^{2}+\left\|\nabla^{k+1} \mathbf{P}\right\|^{2}\right) .
\end{aligned}
$$

Then, combining relations (85)-(87) yields

$$
\begin{aligned}
\lambda_{1} \| & \nabla \nabla^{k} v \|^{2}+\frac{d}{d t}\left\langle\nabla \nabla^{k} v, \nabla^{k} \mathbf{P}\right\rangle \\
= & f(\bar{v})\left\langle\nabla^{k} \mathbf{P}, \nabla \nabla^{k} v\right\rangle \\
& +\left\langle\lambda_{1} \nabla^{k} \operatorname{div} \mathbf{P}-\nabla^{k} F, \nabla^{k} \operatorname{div} \mathbf{P}\right\rangle+\left\langle\nabla \nabla^{k} v, \nabla^{k} G\right\rangle \\
\lesssim & \left\|\nabla^{k} \mathbf{P}\right\|^{2}+\frac{\lambda_{1}}{4}\left\|\nabla \nabla^{k} v\right\|^{2}+\left\|\nabla^{k} \operatorname{div} \mathbf{P}\right\|^{2} \\
& +\delta\left(\left\|\nabla^{k+1} v\right\|^{2}+\left\|\nabla^{k+1} \mathbf{P}\right\|^{2}\right)+\left\langle\nabla \nabla^{k} v, \nabla^{k} G\right\rangle .
\end{aligned}
$$

Similarly, for $\left\langle\nabla \nabla^{k} \mathbf{P}, \nabla^{k} G\right\rangle$, it also holds that

$$
\left\langle\nabla \nabla^{k} \mathbf{P}, \nabla^{k} G\right\rangle \leqslant \delta\left(\left\|\nabla^{k+1} v\right\|^{2}+\left\|\nabla^{k+1} \mathbf{P}\right\|^{2}\right) .
$$

Putting (89) into (88) and summing up for $k=1$ to 2 , we obtain

$$
\frac{d}{d t} \sum_{1 \leq k \leq 2}\left\langle\nabla \nabla^{k} v, \nabla^{2} \mathbf{P}\right\rangle+\left\|\nabla^{2} v\right\|_{1}^{2} \lesssim\|\nabla \mathbf{P}\|_{2}^{2} .
$$

Therefore, multiplying (84) by a sufficiently large positive constant $D_{2}$ and adding it to (90), we can obtain (65) since $\delta>0$ is small enough. Therefore, we have completed the proof of Lemma 6.

\section{Global Existence and Decay Rates}

In this section, we devote ourselves to prove Proposition 3. To begin with, we give the following lemma which will be used later.

Lemma 7. It holds that

$$
\begin{aligned}
& \|\nabla(v, \mathbf{P})(t)\| \lesssim K_{0}(1+t)^{-(3 / 2)(1 / p-1 / 2)-1 / 2} \\
& +\delta \int_{0}^{t}(1+t-\tau)^{-(3 / 2)(1 / p-1 / 2)-1 / 2} \\
& \quad\|\nabla(v, \mathbf{P})(\tau)\|_{2} d \tau
\end{aligned}
$$

for any $0 \leq t \leq T$, where $K_{0}=\left\|v_{0}-\bar{v}\right\|_{H^{3} \cap L^{p}}+\left\|\mathbf{P}_{0}\right\|_{H^{3} \cap L^{3 p /(3-p)}}$ is finite due to the assumptions of Proposition 3. 
Proof. Applying (36)-(37) with $k=1$ and using Duhamel's principle, we have

$$
\begin{aligned}
& \|\nabla(v, \mathbf{P})(t)\| \lesssim K_{0}(1+t)^{-(3 / 2)(1 / p-1 / 2)-1 / 2} \\
& \quad+\int_{0}^{t}(1+t-\tau)^{-(3 / 2)(1 / p-1 / 2)-1 / 2} \\
& \cdot\|(F, G)(\tau)\|_{H^{1} \cap L^{1}} d \tau .
\end{aligned}
$$

From (53), (56), Lemmas A.1 and A.4, and Hölder inequality, we can estimate the nonlinear source terms as follows:

$$
\begin{aligned}
& \|(F, G)(t)\|_{L^{1}} \lesssim \delta\|\nabla(v, \mathbf{P})(t)\|_{1}, \\
& \|(F, G)(t)\|_{1} \leqslant \delta\|\nabla(v, \mathbf{P})(t)\|_{2} .
\end{aligned}
$$

Substituting these estimates into (92) gives (91) and thus proves Lemma 7.

Now, we are in a position to prove Proposition 3.

Proof of Proposition 3. The proof involves the following two steps.

Step 1 . Since $\delta>0$ is suitably small, from Lemmas 5 and 6 , we can choose a suitably large positive constant $D_{3}$ such that

$$
\begin{aligned}
& \frac{d}{d t}\left(D _ { 3 } \left(\|(v, \mathbf{P})\|_{3}^{2}\right.\right. \\
& \left.\quad+\sum_{1 \leq k \leq 3} \int_{\mathbb{R}^{3}} \frac{\chi}{\lambda_{1}-\chi}\left|\nabla^{k} v\right|^{2}+\frac{1}{\lambda}\left(\sigma^{\prime}(v+\bar{v})-\sigma^{\prime}(\bar{v})\right)\left|\nabla^{k} \mathbf{P}\right|^{2} d x\right) \\
& \left.\quad+\sum_{1 \leq k \leq 2}\left\langle\nabla \nabla^{k} v, \nabla^{k} \mathbf{P}\right\rangle(t)\right)+C\left(\|\nabla v(t)\|_{2}^{2}+\|\mathbf{P}(t)\|_{3}^{2}\right) \\
& \quad \leq \delta\|\nabla v(t)\|^{2}
\end{aligned}
$$

Due to (53), it is clear that the expression under $d / d t$ in (94) is equivalent to $\|(v, \mathbf{P})(t)\|_{3}^{2}$. Hence, by integrating (94) directly in time, we obtain (24).

Step 2. Define the temporal energy functional

$$
\begin{aligned}
\mathscr{E}(t) & =D_{2}\left(\|\nabla(v, \mathbf{P})\|_{2}^{2}+\sum_{1 \leq k \leq 3} \int_{\mathbb{R}^{3}} \frac{\chi}{\lambda_{1}-\chi}\left|\nabla^{k} v\right|^{2}\right. \\
+ & \left.\frac{1}{\lambda}\left(\sigma^{\prime}(v+\bar{v})-\sigma^{\prime}(\bar{v})\right)\left|\nabla^{k} \mathbf{P}\right|^{2} d x\right) \\
+ & \sum_{1 \leq k \leq 2}\left\langle\nabla \nabla^{k} v, \nabla^{k} \mathbf{P}\right\rangle,
\end{aligned}
$$

for any $0 \leq t \leq T$, where $\mathscr{E}(t)$ is equivalent to $\|\nabla(v, \mathbf{P})\|_{2}^{2}$ since $D_{2}$ can be large enough.

Due to Lemma 6, we have

$$
\frac{d}{d t} \mathscr{E}(t)+C\left(\left\|\nabla^{2} v\right\|_{1}^{2}+\|\nabla \mathbf{P}\|_{2}^{2}\right) \leqslant \delta\|\nabla v(t)\|^{2} .
$$

Adding $\|\nabla v(t)\|^{2}$ to both sides of (96) gives

$$
\frac{d}{d t} \mathscr{E}(t)+D_{4} \mathscr{E}(t) \leqslant\|\nabla v(t)\|^{2},
$$

where $D_{4}$ is a positive constant independent of $\delta$. Setting

$$
\mathscr{H}(t)=\sup _{0 \leq \tau \leq t}(1+\tau)^{3(1 / p-1 / 2)+1} \mathscr{E}(\tau),
$$

then

$$
\begin{aligned}
\|\nabla(v, \mathbf{P})(\tau)\|_{2} & \leq \sqrt{\mathscr{E}(\tau)} \\
& \leq(1+\tau)^{-(3 / 2)(1 / p-1 / 2)-1 / 2} \sqrt{\mathscr{H}(t)}, \\
0 & \leq \tau \leq t \leq T .
\end{aligned}
$$

This together with (91) yields that

$$
\begin{aligned}
\| & \nabla(v, \mathbf{P})(t) \| \lesssim K_{0}(1+t)^{-(3 / 2)(1 / p-1 / 2)-1 / 2} \\
& +\delta \int_{0}^{t}(1+t-\tau)^{-(3 / 2)(1 / p-1 / 2)-1 / 2} \\
& (1+\tau)^{-(3 / 2)(1 / p-1 / 2)-1 / 2} \sqrt{\mathscr{H}(t)} d \tau \lesssim(1 \\
& +t)^{-(3 / 2)(1 / p-1 / 2)-1 / 2}\left(K_{0}+\delta \sqrt{\mathscr{H}(t)}\right) .
\end{aligned}
$$

Thus, from Gronwall's inequality, (97), and (100), we have

$$
\begin{aligned}
\mathscr{E}(t) & \leq \mathscr{E}(0) e^{-D_{4} t}+\int_{0}^{t} e^{-D_{4}(t-\tau)}\|\nabla v(\tau)\|^{2} d \tau \\
& \leq \mathscr{E}(0) e^{-D_{4} t}+\left(K_{0}^{2}+\delta^{2} \mathscr{H}(t)\right) \\
& \cdot \int_{0}^{t} e^{-D_{4}(t-\tau)}(1+\tau)^{-3(1 / p-1 / 2)-1} d \tau \\
& \leq(1+t)^{-3(1 / p-1 / 2)-1}\left(\mathscr{E}(0)+K_{0}^{2}+\delta^{2} \mathscr{H}(t)\right) .
\end{aligned}
$$

Since $\mathscr{H}$ is nondecreasing, from (101), we have

$$
\mathscr{H}(t) \lesssim\left(\mathscr{E}(0)+K_{0}^{2}+\delta^{2} \mathscr{H}(t)\right),
$$

for any $0 \leq t \leq T$, which together with the smallness of $\delta$ gives that

$$
\mathscr{H}(t) \lesssim\left(\mathscr{E}(0)+K_{0}^{2}\right) \lesssim K_{0}^{2} .
$$

Therefore, (99) and (103) lead to

$$
\begin{aligned}
\|\nabla(v, \mathbf{P})(t)\|_{2} \leq K_{0}(1+t)^{-(3 / 2)(1 / p-1 / 2)-1 / 2}, & \\
& 0 \leq t \leq T
\end{aligned}
$$

and this proves (28). 
Next, we turn to the proofs of (25)-(27). Applying (36)(37) with $k=0$, we have from Duhamel's principle that

$$
\begin{aligned}
& \|v(t)\| \lesssim K_{0}(1+t)^{-(3 / 2)(1 / p-1 / 2)} \\
& +\int_{0}^{t}(1+t-\tau)^{-(3 / 2)(1 / p-1 / 2)} \\
& \cdot\|(F, G)(\tau)\|_{L^{2} \cap L^{1}} d \tau \lesssim K_{0}(1+t)^{-(3 / 2)(1 / p-1 / 2)} \\
& +\delta \int_{0}^{t}(1+t-\tau)^{-(3 / 2)(1 / p-1 / 2)}\|\nabla(v, \mathbf{P})(\tau)\|_{1} d \tau \\
& \lesssim K_{0}(1+t)^{-(3 / 2)(1 / p-1 / 2)} \\
& +K_{0} \delta \int_{0}^{t}(1+t-\tau)^{-(3 / 2)(1 / p-1 / 2)} \\
& \cdot(1+\tau)^{-(3 / 2)(1 / p-1 / 2)-1 / 2} d \tau \lesssim K_{0}(1 \\
& +t)^{-(3 / 2)(1 / p-1 / 2)} \text {, } \\
& \|\mathbf{P}(t)\| \lesssim K_{0}(1+t)^{-(3 / 2)(1 / p-1 / 2)-1 / 2} \\
& +\int_{0}^{t}(1+t-\tau)^{-(3 / 2)(1 / p-1 / 2)-1 / 2} \\
& \cdot\|(F, G)(\tau)\|_{L^{2} \cap L^{1}} d \tau \lesssim K_{0}(1 \\
& +t)^{-(3 / 2)(1 / p-1 / 2)-1 / 2} \\
& +\delta \int_{0}^{t}(1+t-\tau)^{-(3 / 2)(1 / p-1 / 2)-1 / 2} \\
& \cdot\|\nabla(v, \mathbf{P})(\tau)\|_{1} d \tau \lesssim K_{0}(1 \\
& +t)^{-(3 / 2)(1 / p-1 / 2)-1 / 2} \\
& +K_{0} \delta \int_{0}^{t}(1+t-\tau)^{-(3 / 2)(1 / p-1 / 2)-1 / 2} \\
& \cdot(1+\tau)^{-(3 / 2)(1 / p-1 / 2)-1 / 2} d \tau \lesssim K_{0}(1 \\
& +t)^{-(3 / 2)(1 / p-1 / 2)-1 / 2},
\end{aligned}
$$

for any $0 \leq t \leq T$. On the other hand, from Lemma A.1 and (29), we also have

$$
\begin{aligned}
\|(v, \mathbf{P})(t)\|_{L^{q}} & \leqslant\|\nabla(v, \mathbf{P})(t)\|_{1} \\
& \leqslant K_{0}(1+t)^{-(3 / 2)(1 / p-1 / 2)-1 / 2}, \\
& \quad 6 \leq q \leq \infty .
\end{aligned}
$$

Thus, we can deduce (25)-(27) from the interpolation and (105)-(106) immediately.

Finally, we prove (29). By virtue of (18) and (28), we have

$$
\begin{aligned}
\left\|\partial_{t}(v, \mathbf{P})(t)\right\| & \lesssim\|\mathbf{P} \cdot \nabla v\|+\|\operatorname{div} \mathbf{P}\|+\|\nabla v\|+\|\mathbf{P}\| \\
& \lesssim\|\nabla v\|+\|\mathbf{P}\|_{1} \\
& \lesssim K_{0}(1+t)^{-(3 / 2)(1 / p-1 / 2)-1 / 2}
\end{aligned}
$$

for any $0 \leq t \leq T$. Therefore, (29) is proved and therefore we have completed the proof of Proposition 3.

\section{Appendix}

\section{Analytic Tools}

We will extensively use the Sobolev interpolation of the Gagliardo-Nirenberg inequality.

Lemma A.1. Let $0 \leq i, j \leq k$; then one has

$$
\left\|\nabla^{i} f\right\|_{L^{p}} \lesssim\left\|\nabla^{j} f\right\|_{L^{q}}^{1-\theta}\left\|\nabla^{k} f\right\|_{L^{r}}^{\theta},
$$

where $\theta$ satisfies

$$
\frac{i}{3}-\frac{1}{p}=\left(\frac{j}{3}-\frac{1}{q}\right)(1-\theta)+\left(\frac{k}{3}-\frac{1}{r}\right) \theta .
$$

Proof. This is a special case of [33, pp. 125, Theorem].

Next, we recall the following product estimate.

Lemma A.2. Let $k \geq 1$ be an integer; then it holds that

$$
\left\|\nabla^{k}(f g)\right\|_{L^{p}} \lesssim\|f\|_{L^{p_{1}}}\left\|\nabla^{k} g\right\|_{L^{p_{2}}}+\left\|\nabla^{k} f\right\|_{L^{p_{3}}}\|g\|_{L^{p_{4}}},
$$

where $p, p_{2}, p_{3} \in(1,+\infty)$ and

$$
\frac{1}{p}=\frac{1}{p_{1}}+\frac{1}{p_{2}}=\frac{1}{p_{3}}+\frac{1}{p_{4}} .
$$

Proof. For $p=p_{2}=p_{3}=2$, it can be proved by using Lemma A.1. For the general cases, one may refer to [51, Lemma 3.1].

We also need the following commutator estimate.

Lemma A.3. Let $k \geq 1$ be an integer and define the commutator

$$
\left[\nabla^{k}, f\right] g=\nabla^{k}(f g)-f \nabla^{k} g
$$

Then one has

$$
\begin{aligned}
\left\|\left[\nabla^{k}, f\right] g\right\|_{L^{p}} \lesssim & \|\nabla f\|_{L^{p_{1}}}\left\|\nabla^{k-1} g\right\|_{L^{p_{2}}} \\
& +\left\|\nabla^{k} f\right\|_{L^{p_{3}}}\|g\|_{L^{p_{4}}},
\end{aligned}
$$

where $p, p_{2}, p_{3} \in(1,+\infty)$ and

$$
\frac{1}{p}=\frac{1}{p_{1}}+\frac{1}{p_{2}}=\frac{1}{p_{3}}+\frac{1}{p_{4}} .
$$

Proof. The proof can be found in [51, Lemma 3.1].

Finally, to estimate the $L^{p}$-norm of the spatial derivatives of $f(v+\bar{v})-f(\bar{v}), h(v)$, and $\sigma^{\prime}(v+\bar{v})-\sigma^{\prime}(\bar{v})$, we shall need the technique lemma. 
Lemma A.4. Assume that $\|\varrho\|_{L^{\infty}} \leq 1$. Let $g(\varrho)$ be a smooth function of $\varrho$ with bounded derivatives of any order, then, for any integer $m \geq 1$ and $1 \leq p \leq \infty$, one has

$$
\left\|\nabla^{m}(g(\varrho))\right\|_{L^{p}} \lesssim\left\|\nabla^{m} \varrho\right\|_{L^{p}} .
$$

Proof. Notice that, for $m \geq 1$,

$$
\begin{aligned}
\nabla^{m} & (g(\varrho)) \\
& =\text { a sum of products } g^{\gamma_{1}, \ldots, \gamma_{n}}(\varrho) \nabla^{\gamma_{1}} \varrho \cdots \nabla^{\gamma_{n}} \varrho,
\end{aligned}
$$

where the functions $g^{\gamma_{1}, \ldots, \gamma_{n}}(\varrho)$ are some derivatives of $g(\varrho)$ and $1 \leq \gamma_{i} \leq m, i=1, \ldots, n$, with $\gamma_{1}+\cdots+\gamma_{n}=m$. We then use the Sobolev interpolation of Lemma A.1 to bound

$$
\begin{aligned}
& \left\|\nabla^{m}(g(\varrho))\right\|_{L^{P}} \lesssim\left\|\nabla^{\gamma_{1}} \varrho\right\|_{L^{p m / \gamma_{1}}} \cdots\left\|\nabla^{\gamma_{n}} \varrho\right\|_{L^{p m / \gamma_{n}}} \\
& \quad \lesssim\|\varrho\|_{L^{\infty}}^{1-\gamma_{1} / m}\left\|\nabla^{m} \varrho\right\|_{L^{p}}^{\gamma_{1} / m} \cdots\|\varrho\|_{L^{\infty}}^{1-\gamma_{n} / m}\left\|\nabla^{m} \varrho\right\|_{L^{p}}^{\gamma_{n} / m} \\
& \quad \lesssim\|\varrho\|_{L^{\infty}}^{n-1}\left\|\nabla^{m} \varrho\right\|_{L^{p}} .
\end{aligned}
$$

Hence, we conclude our lemma since $\|\varrho\|_{L^{\infty}} \leq 1$.

\section{Conflicts of Interest}

The authors declare that there are no conflicts of interest regarding the publication of this article.

\section{Acknowledgments}

This work was partially supported by the Hunan Provincial Natural Science Foundation of China no. 2017JJ2105, the National Natural Science Foundation of China no. 11571280, no. 11301172, and no. 11226170, and National Scholarship Fund in Hunan Province Cooperation Projects.

\section{References}

[1] K. Saxton, R. Saxton, and W. Kosinski, "On second sound at the critical temperature," Quarterly of Applied Mathematics, vol. 57, no. 4, pp. 723-740, 1999.

[2] K. Saxton and R. Saxton, "Nonlinearity and memory effects in low temperature heat propagation," Archives of Mechanics, vol. 52, no. 1, pp. 127-142, 2000.

[3] K. Saxton and R. Saxton, "Phase transitions and change of type in low-temperature heat propagation," SIAM Journal on Applied Mathematics, vol. 66, no. 5, pp. 1689-1702, 2006.

[4] K. Saxton and R. Saxton, "On the influence of damping in hyperbolic equations with parabolic degeneracy," Quarterly of Applied Mathematics, vol. 70, no. 1, pp. 171-180, 2012.

[5] H. Li and K. Saxton, "Asymptotic behavior of solutions to quasilinear hyperbolic equations with nonlinear damping," Quarterly of Applied Mathematics, vol. 61, no. 2, pp. 295-313, 2003.

[6] S. F. Geng and L. N. Zhang, " $L^{p}$-convergence rates to nonlinear diffusion waves for quasilinear equations with nonlinear damping," Zeitschrift für angewandte Mathematik und Physik, vol. 66, no. 1, pp. 31-50, 2015.
[7] S. Geng and L. Zhang, "Boundary effects and large-time behaviour for quasilinear equations with nonlinear damping," Proceedings of the Royal Society of Edinburgh. Section A. Mathematics, vol. 145, no. 5, pp. 959-978, 2015.

[8] S. Geng and Z. Wang, "Convergence rates to nonlinear diffusion waves for solutions to the system of compressible adiabatic flow through porous media," Communications in Partial Differential Equations, vol. 36, no. 5, pp. 850-872, 2011.

[9] L. Hsiao, Quasilinear Hyperbolic Systems and Dissipative Mechanisms, World Scientific, Singapore, 1998.

[10] L. Hsiao and T.-P. Liu, "Convergence to nonlinear diffusion waves for solutions of a system of hyperbolic conservation laws with damping," Communications in Mathematical Physics, vol. 143, no. 3, pp. 599-605, 1992.

[11] L. Hsiao and T. P. Liu, "Nonlinear diffusive phenomena of nonlinear hyperbolic systems," Chinese Annals of Mathematics, Series B, vol. 14, pp. 1-16, 1993.

[12] L. Hsiao and T. Luo, "Nonlinear diffusive phenomena of solutions for the system of compressible adiabatic flow through porous media," Journal of Differential Equations, vol. 125, no. 2, pp. 329-365, 1996.

[13] L. Hsiao and R. Pan, "Initial-boundary value problem for the system of compressible adiabatic flow through porous media," Journal of Differential Equations, vol. 159, no. 1, pp. 280-305, 1999.

[14] L. Hsiao and R. H. Pan, "The damped p-system with boundary effects," Contemporary Mathematics, vol. 255, pp. 109-123, 2000.

[15] L. Hsiao and D. Serre, "Global existence of solutions for the system of compressible adiabatic flow through porous media," SIAM Journal on Mathematical Analysis, vol. 27, no. 1, pp. 70-77, 1996.

[16] F. Huang, P. Marcati, and R. Pan, "Convergence to the Barenblatt solution for the compressible Euler equations with damping and vacuum," Archive for Rational Mechanics and Analysis, vol. 176, no. 1, pp. 1-24, 2005.

[17] F. Huang and R. Pan, "Convergence rate for compressible Euler equations with damping and vacuum," Archive for Rational Mechanics and Analysis, vol. 166, no. 4, pp. 359-376, 2003.

[18] F. Huang and R. Pan, "Asymptotic behavior of the solutions to the damped compressible Euler equations with vacuum," Journal of Differential Equations, vol. 220, no. 1, pp. 207-233, 2006.

[19] M. N. Jiang, L. Z. Ruan, and J. Zhang, "Existence of global smooth solution to the initial boundary value problem for $p$ system with damping," Nonlinear Analysis, vol. 70, no. 6, pp. 2471-2479, 2009.

[20] M. Jiang and Y. Zhang, "Existence and asymptotic behavior of global smooth solution for $p$-system with nonlinear damping and fixed boundary effect," Mathematical Methods in the Applied Sciences, vol. 37, no. 17, pp. 2585-2596, 2014.

[21] M. N. Jiang and C. J. Zhu, "Convergence rates to nonlinear diffusion waves for $p$-system with nonlinear damping on quadrant," Discrete and Continuous Dynamical Systems, vol. 23, no. 3, pp. 887-918, 2009.

[22] M. N. Jiang and C. J. Zhu, "Convergence to strong nonlinear diffusion waves for solutions to $p$-system with damping on quadrant," Journal of Differential Equations, vol. 246, no. 1, pp. 50-77, 2009.

[23] C. K. Lin, C. T. Lin, and M. Mei, "Asymptotic behavior of solution to nonlinear damped p-system with boundary effect," International Journal of Numerical Analysis and Modeling, Series $B$, vol. 1, no. 1, pp. 70-92, 2010. 
[24] T.-P. Liu, "Compressible flow with damping and vacuum," Japan Journal of Industrial and Applied Mathematics, vol. 13, no. 1, pp. 25-32, 1996.

[25] H. Ma and M. Mei, "Best asymptotic profile for linear damped p-system with boundary effect," Journal of Differential Equations, vol. 249, no. 2, pp. 446-484, 2010.

[26] P. Marcati and M. Mei, "Convergence to nonlinear diffusion waves for solutions of the initial boundary problem to the hyperbolic conservation laws with damping," Quarterly of Applied Mathematics, vol. 58, no. 4, pp. 763-784, 2000.

[27] P. Marcati, M. Mei, and B. Rubino, "Optimal convergence rates to diffusion waves for solutions of the hyperbolic conservation laws with damping," Journal of Mathematical Fluid Mechanics, vol. 7, supplement 2, pp. S224-S240, 2005.

[28] P. Marcati and A. Milani, “The one-dimensional Darcy's law as the limit of a compressible Euler flow," Journal of Differential Equations, vol. 84, no. 1, pp. 129-147, 1990.

[29] P. Marcati and B. Rubino, "Hyperbolic to parabolic relaxation theory for quasilinear first order systems," Journal of Differential Equations, vol. 162, no. 2, pp. 359-399, 2000.

[30] P. Marcati and R. Pan, "On the diffusive profiles for the system of compressible adiabatic flow through porous media," SIAM Journal on Mathematical Analysis, vol. 33, no. 4, pp. 790-826, 2001.

[31] M. Mei, "Nonlinear diffusion waves for hyperbolic $p$-system with nonlinear damping," Journal of Differential Equations, vol. 247, no. 4, pp. 1275-1296, 2009.

[32] M. Mei, "Best asymptotic profile for hyperbolic $p$-system with damping," SIAM Journal on Mathematical Analysis, vol. 42, no. 1, pp. 1-23, 2010.

[33] L. Nirenberg, "On elliptic partial differential equations," Annali della Scuola Normale Superiore di Pisa, vol. 13, pp. 115-162, 1959.

[34] R. Pan, "Boundary effects and large time behavior for the system of compressible adiabatic flow through porous media," Michigan Mathematical Journal, vol. 49, no. 3, pp. 519-540, 2001.

[35] R. Pan and K. Zhao, "The 3D compressible Euler equations with damping in a bounded domain," Journal of Differential Equations, vol. 246, no. 2, pp. 581-596, 2009.

[36] T. C. Sideris, B. Thomases, and D. Wang, "Long time behavior of solutions to the 3D compressible Euler equations with damping," Communications in Partial Differential Equations, vol. 28, no. 3-4, pp. 795-816, 2003.

[37] Z. Tan and Y. Wang, "Global solution and large-time behavior of the 3D compressible Euler equations with damping," Journal of Differential Equations, vol. 254, no. 4, pp. 1686-1704, 2013.

[38] Z. Tan and G. C. Wu, "Large time behavior of solutions for compressible Euler equations with damping in $\mathbb{R}^{3}$," Journal of Differential Equations, vol. 252, no. 2, pp. 1546-1561, 2012.

[39] W. Wang and T. Yang, "The pointwise estimates of solutions for Euler equations with damping in multi-dimensions," Journal of Differential Equations, vol. 173, no. 2, pp. 410-450, 2001.

[40] G. C. Wu, Z. Tan, and J. Huang, "Global existence and large time behavior for the system of compressible adiabatic flow through porous media in $\mathbb{R}^{3}$," Journal of Differential Equations, vol. 255, no. 5, pp. 865-880, 2013.

[41] Y. H. Zhang and Z. Tan, "Existence and asymptotic behavior of global smooth solution for $p$-system with damping and boundary effect," Nonlinear Analysis: Theory, Methods \& Applications, vol. 72, no. 5, pp. 2499-2513, 2010.

[42] Y. Zhang and G. Wu, "Global existence and asymptotic behavior for the 3D compressible non-isentropic Euler equations with damping," Acta Mathematica Scientia, vol. 34, no. 2, pp. 424434, 2014.

[43] Y. Zhang and G. Wu, "The 3D non-isentropic compressible Euler equations with damping in a bounded domain," Chinese Annals of Mathematics. Series B, vol. 37, no. 6, pp. 915-928, 2016.

[44] C. J. Zhu, "Convergence rates to nonlinear diffusion waves for weak entropy solutions to $p$-system with damping," Science in China, Series A: Mathematics, vol. 46, no. 4, pp. 562-575, 2003.

[45] C. Zhu and M. Jiang, " $L^{p}$-decay rates to nonlinear diffusion waves for $p$-system with nonlinear damping," Science in China, Series A: Mathematics, vol. 49, no. 6, pp. 721-739, 2006.

[46] H. J. Zhao, "Convergence to strong nonlinear diffusion waves for solutions of $p$-system with damping," Journal of Differential Equations, vol. 174, no. 1, pp. 200-236, 2001.

[47] Y. H. Zhang and C. J. Zhu, "Global existence and optimal convergence rates for the strong solutions in $\mathrm{H}^{2}$ to the $3 \mathrm{D}$ viscous liquid-gas two-phase flow model," Journal of Differential Equations, vol. 258, no. 7, pp. 2315-2338, 2015.

[48] Y. H. Zhang, "Decay of the 3D inviscid liquid-gas two-phase flow model," Zeitschrift für Angewandte Mathematik und Physik, vol. 67, article 54, 2016.

[49] A. Matsumura and T. Nishida, "The initial value problem for the equations of motion of compressible viscous and heatconductive fluids," Proceedings of the Japan Academy, Series A: Mathematical Sciences, vol. 55, no. 9, pp. 337-342, 1979.

[50] T. Nishida, Nonlinear Hyperbolic Equations and Related Topics in Fluid Dynamics, Publications Mathématiques d'Orsay, 1978.

[51] N. Ju, "Existence and uniqueness of the solution to the dissipative 2D quasi-geostrophic equations in the Sobolev space," Communications in Mathematical Physics, vol. 251, no. 2, pp. 365-376, 2004. 


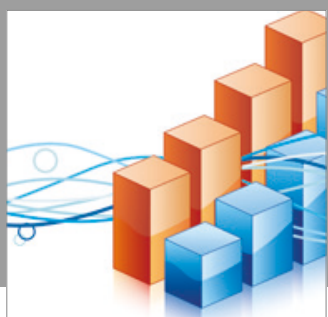

Advances in

Operations Research

vatersals

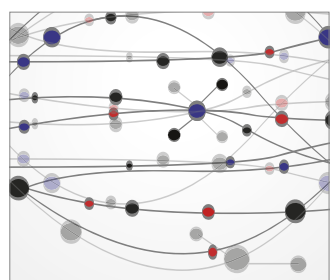

\section{The Scientific} World Journal
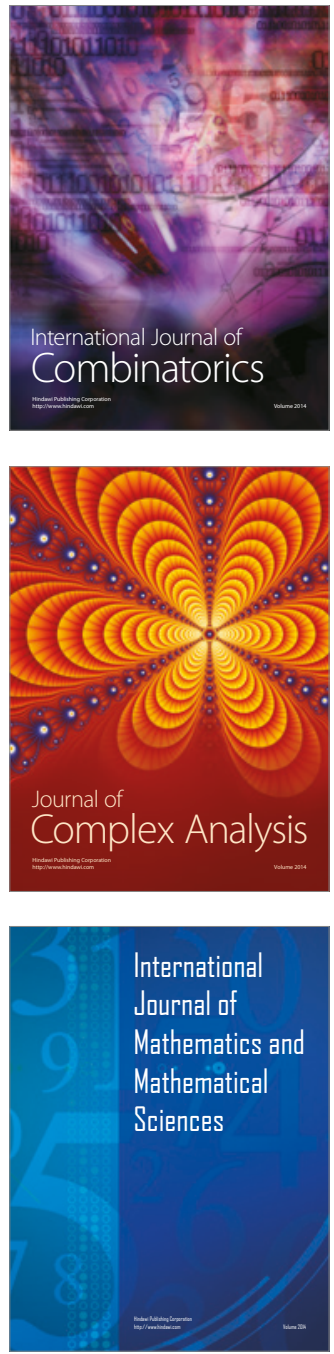
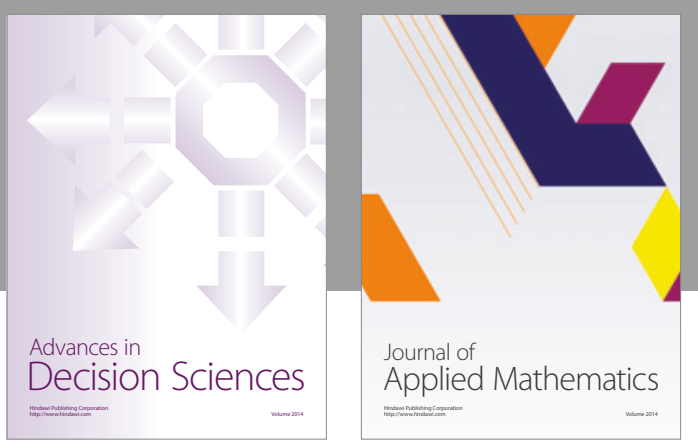

Algebra

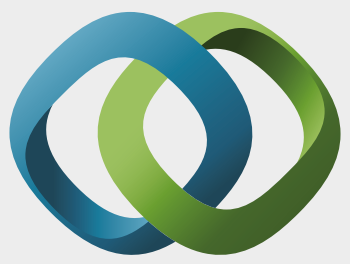

\section{Hindawi}

Submit your manuscripts at

https://www.hindawi.com
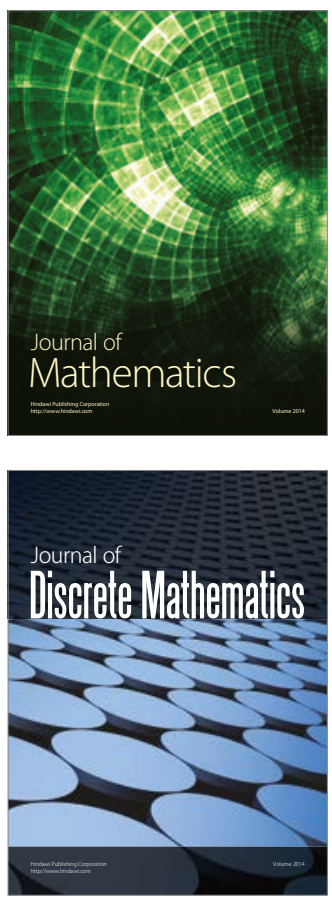

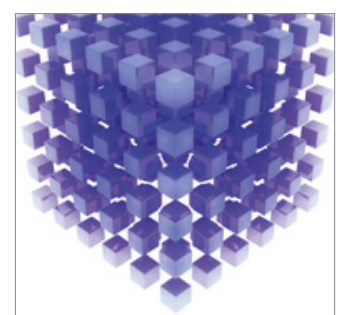

Mathematical Problems in Engineering
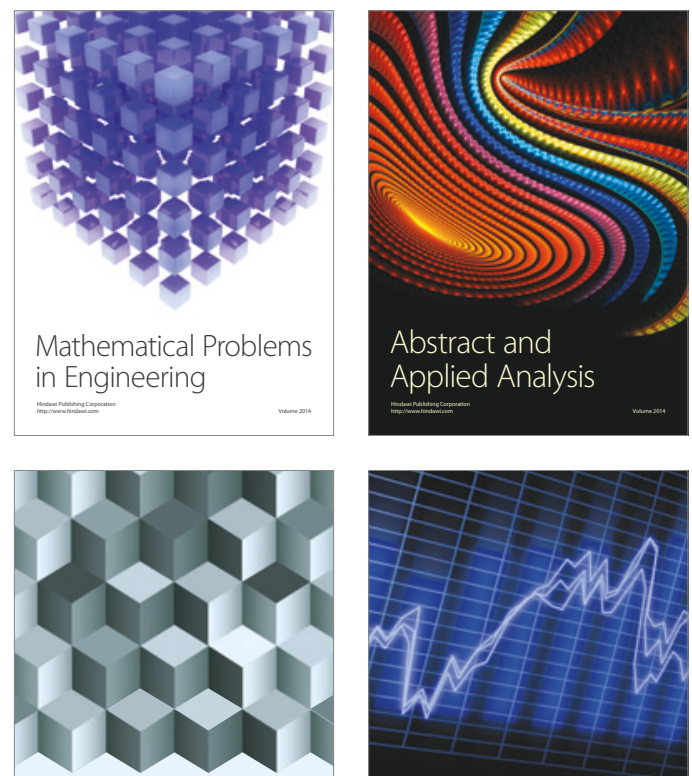

Journal of

Function Spaces

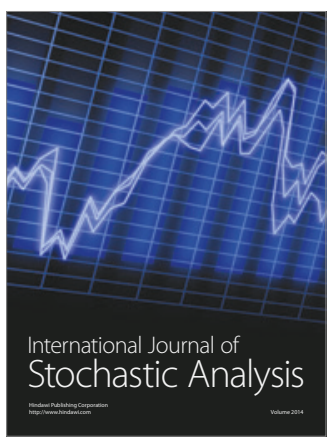

Probability and Statistics
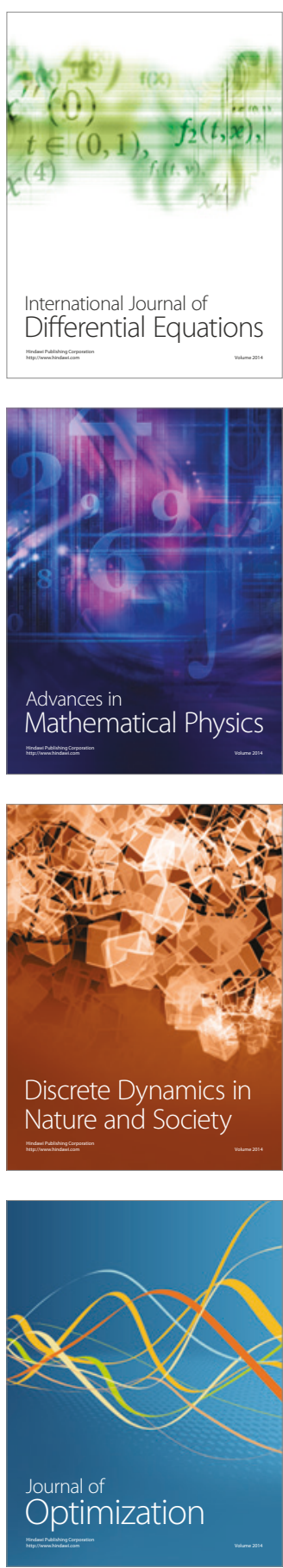